\title{
LOCAL WELL-POSEDNESS FOR BOLTZMANN'S EQUATION AND THE BOLTZMANN HIERARCHY VIA WIGNER TRANSFORM
}

\author{
THOMAS CHEN, RYAN DENLINGER, AND NATAŠA PAVLOVIĆ
}

\begin{abstract}
We use the dispersive properties of the linear Schrödinger equation to prove local well-posedness results for the Boltzmann equation and the related Boltzmann hierarchy, set in the spatial domain $\mathbb{R}^{d}$ for $d \geq 2$. The proofs are based on the use of the (inverse) Wigner transform along with the spacetime Fourier transform. The norms for the initial data $f_{0}$ are weighted versions of the Sobolev spaces $L_{v}^{2} H_{x}^{\alpha}$ with $\alpha \in\left(\frac{d-1}{2}, \infty\right)$. Our main results are local well-posedness for the Boltzmann equation for cutoff Maxwell molecules and hard spheres, as well as local well-posedness for the Boltzmann hierarchy for cutoff Maxwell molecules (but not hard spheres); the latter result holds without any factorization assumption for the initial data.
\end{abstract}

\section{INTRODUCTION}

Boltzmann's equation is an evolutionary partial differential equation (PDE) which describes the behavior of a dilute gas of identical particles in a specific scaling limit. The equation describes the time evolution of a density function $f(t, x, v) \geq 0$, where $x, v \in \mathbb{R}^{d}$ are the position and velocity of a typical particle.

The Cauchy problem for Boltzmann's equation is one of the fundamental mathematical problems in kinetic theory and it may be written in the following form:

$$
\begin{aligned}
& \left(\partial_{t}+v \cdot \nabla_{x}\right) f(t, x, v)=Q(f, f)(t, x, v) \\
& f(0, x, v)=f_{0}(x, v)
\end{aligned}
$$

where the collision operator $Q$ is defined as follows:

$$
\begin{aligned}
Q(f, f)(t, x, v)= & \int_{\mathbb{R}^{d}} \int_{\mathbb{S}^{d-1}} d \omega d v_{2} \mathbf{b}\left(\left|v-v_{2}\right|, \omega \cdot \frac{v-v_{2}}{\left|v-v_{2}\right|}\right) \times \\
& \times\left(f\left(t, x, v^{*}\right) f\left(t, x, v_{2}^{*}\right)-f(t, x, v) f\left(t, x, v_{2}\right)\right)
\end{aligned}
$$

The collision kernel $\mathbf{b}$ is a function which depends on the physical interaction between particles; pre-collisional and post-collisional velocities are related by the following involutive transformation, for $v, v_{2} \in \mathbb{R}^{d}$ and fixed $\omega \in \mathbb{S}^{d-1}$ :

$$
\begin{aligned}
& v^{*}=v+\left(\omega \cdot\left(v_{2}-v\right)\right) \omega \\
& v_{2}^{*}=v_{2}-\left(\omega \cdot\left(v_{2}-v\right)\right) \omega
\end{aligned}
$$


The most general known solutions of Boltzmann's equation are the renormalized solutions of [6], which exist globally in time for arbitrary data $f_{0}$ having finite mass, second moments and entropy:

$$
\int_{\mathbb{R}^{d} \times \mathbb{R}^{d}} f_{0}(x, v)\left(1+|v|^{2}+|x|^{2}+\log \left(1+f_{0}(x, v)\right)\right) d x d v<+\infty .
$$

However, renormalized solutions have many limitations; for instance, they are not known to solve the Boltzmann equation in the usual distributional sense (which makes them difficult to manipulate), nor are they known to be unique, nor are they known to conserve energy. A different and very fruitful line of investigation considers solutions close to an equilibrium distribution of fixed temperature, see e.g. [1,7, 12, 13, 19, 20]. These solutions exist globally in time and enjoy uniqueness and continuous dependence in appropriate functional spaces; however, the theory only applies in a small neighborhood of equilibrium.

Henceforth we will not concern ourselves with the (very difficult) problem of global well-posedness for Boltzmann's equation. Instead we will be interested in the local theory of well-posedness. Generally this means we want to prove existence, uniqueness and continuous dependence of solutions, locally in time and for large data, with regularity as low as possible. See [2, 3, 15] for some existing theories of local solutions for Boltzmann's equation. We especially refer to Remark 1 of of [2], which provides (in the case of Grad cut-off) a large data local well-posedness result which parallels our Theorem 2.2 when $\alpha>\frac{d}{2}$ in $d=31$ Our main intention, however, is not to investigate optimal regularity spaces for solving Boltzmann's equation. Rather, we intend to demonstrate the close connection between Boltzmann's equation and nonlinear Schrödinger equations (NLS) in the density matrix formulation; this connection has been recognized implicitly for some time, but we wish to make it quite explicit and to the best of our knowledge this is the first time such an explicit connection has been established 2 The local well-posedness theory for NLS is by now very mature and it is our hope that some tools which have been useful for NLS will turn out to be applicable to the corresponding problem for Boltzmann's equation. If the theory can be made precise enough, it may turn out to be useful for such problems as global well-posedness or the derivation of Boltzmann's equation from deterministic particle systems.

Besides providing a new approach to proving local well-posedness for Boltzmann's equation, we will also prove new results concerning the Boltzmann hierarchy for at least some collision kernels. The Boltzmann hierarchy is an infinite hierarchy of coupled PDE which describes a gas of infinitely

\footnotetext{
${ }^{1}$ We are able to prove a conditional local well-posedness result when $f_{0}$ is in a weighted version of $L_{v}^{2} H_{x}^{\alpha}$ with $\alpha>\frac{d-1}{2}$ (here conditional means that uniqueness only holds assuming some auxiliary estimate satisfied by the constructed solution). It is conceivable that the uniqueness is unconditional when $\alpha>\frac{d}{2}$, cf. [2], but we do not pursue this issue. ${ }^{2}$ We emphasize that we do not make use of any semiclassical limit.
} 
many particles, possibly accounting for correlations between particles. For some class of collisional kernels, the Boltzmann hierarchy appears in the derivation of Boltzmann's equation from classical system of many particles. See e.g. [11, 18]. The connection between the Boltzmann hierarchy and Boltzmann equation lies in the fact that the Boltzmann hierarchy admits a class of factorized solutions with each factor being a solution to the Boltzmann equation.

The classical local well-posedness result for the Boltzmann hierarchy is due to Lanford [18, who assumes $L^{\infty}$ bounds on the initial data. Our results establish local well-posedness in a functional setting much different than Lanford's; in particular, we can work with spaces that do not embed locally into $L^{\infty}$ in any variable. Unfortunately we cannot report any new results concerning the Boltzmann hierarchy for hard spheres; this is the topic of ongoing research.

The idea at the heart of our proofs is to take the inverse Wigner transform of Boltzmann's equation (resp. the Boltzmann hierarchy). The transport operator

$$
\left(\partial_{t}+v \cdot \nabla_{x}\right)
$$

is transformed into the linear Schrödinger operator

$$
\left(i \partial_{t}+\frac{1}{2}\left(\Delta_{x}-\Delta_{x^{\prime}}\right)\right)
$$

and the nonlinear operator $Q(f, f)$ becomes a new operator $B(\gamma, \gamma)$. This puts us in a situation where we can prove a bilinear estimate of the similar type as the one proved in the work of Klainerman and Machedon [17. Subsequently we can employ the iteration method inspired by the one of Chen and Pavlović [5]; these methods were originally devised for proving the local well-posedness of the Gross-Pitaevskii hierarchy. In this paper we implement them at the level of the transformed Boltzmann equation as well as at the level of the transformed Boltzmann hierarchy 3 The main point that we make here is that the transformed Boltzmann equation becomes a nonlinear Schrödinger equation, and the transformed Boltzmann hierarchy becomes a Schrödinger type hierarchy (usually called Gross-Pitaevskii hierarchy) with nonlinearities that encode information about the interaction between particles encoded in the Boltzmann collision kernels. Once we are at the level of such nonlinear Schrödinger equation/hierarchy, we develop tools and emloy techniques for local well-posedness inspired by tools and techniques that have been recently introduced in the context of the GrossPitaevskii hierarchy.

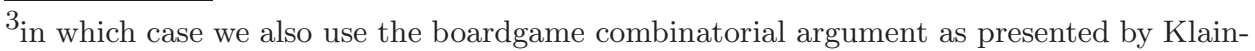
erman and Machedon [17, which is a reformulation of the combinatorial methods of Erdös, Schlein and Yau, 8] 10.
} 
Organization of the paper. Section 2 describes in detail the main results we will prove, using the Wigner transform. Section 3 gives the proof of a crucial proposition which is used to prove all our results, and constitutes the main technical contribution of the paper. Section 4 is devoted to the proof of local well-posedness for the Boltzmann equation; this result extends to cutoff Maxwell molecules, hard spheres, and variable hard sphere models. Section 5 gives a brief outline of the proof of local well-posedness for the Boltzmann hierarchy, including the case of cutoff Maxwell molecules (but not hard spheres).

Acknowledgements. The work of T.C. is supported by NSF grants DMS1151414 (CAREER) and DMS-1262411. R. D. gratefully acknowledges support from a postdoctoral fellowship at the University of Texas at Austin. The work of N.P. is supported by NSF grant DMS-1516228.

\section{Main Results}

2.1. Notation and preliminaries. Given a function $f(x, v) \in L_{x, v}^{2}$ we define its inverse Wigner transform $\gamma\left(x, x^{\prime}\right) \in L_{x, x^{\prime}}^{2}$ by the following formula:

$$
\gamma\left(x, x^{\prime}\right)=\int_{\mathbb{R}^{d}} f\left(\frac{x+x^{\prime}}{2}, v\right) e^{i v \cdot\left(x-x^{\prime}\right)} d v
$$

The inverse of the inverse Wigner transform is the usual Wigner transform, namely:

$$
f(x, v)=\frac{1}{(2 \pi)^{d}} \int_{\mathbb{R}^{d}} \gamma\left(x+\frac{y}{2}, x-\frac{y}{2}\right) e^{-i v \cdot y} d y
$$

All of our main results will be stated in terms of $\gamma$; in particular, if we say $f(t)$ satisfies Boltzmann's equation, we mean that $\gamma(t)$ solves the Duhamel formula associated with the inverse Wigner transform of the Boltzmann equation.

Remark. Note that if $\gamma\left(x, x^{\prime}\right)=\overline{\gamma\left(x^{\prime}, x\right)}$ for all $x, x^{\prime} \in \mathbb{R}^{d}$, then $f$ is everywhere real-valued; the converse also holds. In particular, it is easy to check on the inverse Wigner side that $f$ is real-valued. It is much less simple to determine whether $f$ is non-negative, and this is an issue we do not address in the present work.

Throughout the paper, we will assume that $0 \leq \mathbf{b} \in L_{A}^{\infty}$ for some $A \in$ $[0,1]$, where we have defined

$$
\|\mathbf{b}\|_{L_{A}^{\infty}}=\sup _{u \in \mathbb{R}^{d}, \omega \in \mathbb{S}^{d-1}} \frac{\left|\mathbf{b}\left(|u|, \omega \cdot \frac{u}{|u|}\right)\right|}{1+|u|^{A}}
$$

We will require the Fourier transform of the collision kernel, which is written

$$
\hat{\mathbf{b}}^{\omega}(\xi)=\int_{\mathbb{R}^{d}} \mathbf{b}\left(|u|, \omega \cdot \frac{u}{|u|}\right) e^{-i u \cdot \xi} d u
$$


Note that $\hat{\mathbf{b}}^{\omega}$ is a tempered distribution in general. Special cases include $\mathbf{b} \equiv 1$ with $A=0$ (Maxwell molecules with angular cut-off), $\mathbf{b}=[\omega \cdot u]_{+}$ with $A=1$ (hard spheres), and $0<A<1$ for variable hard sphere models. Not all results will apply for the full range $A \in[0,1]$.

We introduce the weighted Sobolev spaces which define our functional setting. Let $\hat{\gamma}$ denote the Fourier transform of $\gamma$ :

$$
\hat{\gamma}\left(\xi, \xi^{\prime}\right)=\int_{\mathbb{R}^{d} \times \mathbb{R}^{d}} e^{-i x \cdot \xi} e^{-i x^{\prime} \cdot \xi^{\prime}} \gamma\left(x, x^{\prime}\right) d x d x^{\prime}
$$

Then, for any $\alpha, \beta, \kappa \geq 0$, and any $\sigma>0$,

$$
\left\|\gamma\left(x, x^{\prime}\right)\right\|_{H^{\alpha, \beta, \sigma, \kappa}}=\left\|\left\langle\xi+\xi^{\prime}\right\rangle^{\alpha}\left\langle\xi-\xi^{\prime}\right\rangle^{\beta} e^{\kappa\left\langle\xi-\xi^{\prime}\right\rangle^{\frac{1}{\sigma}}} \hat{\gamma}\left(\xi, \xi^{\prime}\right)\right\|_{L_{\xi, \xi^{\prime}}^{2}}
$$

Note that this norm is equivalent to the following norm for the classical densities:

$$
\left\|\langle 2 v\rangle^{\beta} e^{\kappa\langle 2 v\rangle^{\frac{1}{\sigma}}}\left(1-\Delta_{x}\right)^{\frac{\alpha}{2}} f(x, v)\right\|_{L_{x, v}^{2}}
$$

Remark. We emphasize that we can allow $\kappa=0$ for some of our results, e.g. the case of cutoff Maxwell molecules. We always require $\kappa>0$ in the case of hard spheres.

2.2. Warm-up: Free transport. We present a few brief remarks on the free transport equation before turning to our main results. The main point we wish to make is that if $f(t, x, v)$ solves the equation

$$
\left(\partial_{t}+v \cdot \nabla_{x}\right) f=0
$$

then the inverse Wigner transform $\gamma\left(t, x, x^{\prime}\right)$ satisfies the following linear Schrödinger equation:

$$
\left(i \partial_{t}+\frac{1}{2}\left(\Delta_{x}-\Delta_{x^{\prime}}\right)\right) \gamma\left(t, x, x^{\prime}\right)=0
$$

We emphasize that this correspondence does not rely on any semiclassical limit.

Example 2.1. If $\gamma\left(t, x, x^{\prime}\right)=e^{i k \cdot\left(x-x^{\prime}\right)}$ for some $k \in \mathbb{R}^{d}$, then $\gamma$ solves (13) and $f(t, x, v)=c \delta(v-k)$ solves (12).

Example 2.2. If $\gamma\left(t, x, x^{\prime}\right)=|t|^{-d} e^{i\left(|x|^{2}-\left|x^{\prime}\right|^{2}\right) /(2 t)}$, then $\gamma$ solves (13) and $f(t, x, v)=c \delta(x-v t)$ solves (12).

Example 2.3. If $f(t, x, v)=\delta(x-v t) \delta\left(v-v_{0}\right)$, for a fixed $v_{0} \in \mathbb{R}^{d}$, then $f$ solves (12); moreover, the classical state (position and velocity) is known exactly. In any case, $\gamma$ exists as a distribution; for any $u\left(t, x, x^{\prime}\right) \in \mathcal{C}_{0}^{\infty}(\mathbb{R} \times$ 
$\mathbb{R}^{d} \times \mathbb{R}^{d}$ ) we have

$$
\begin{aligned}
& \int_{\mathbb{R}^{\prime} \times \mathbb{R}^{d} \times \mathbb{R}^{d}} \gamma\left(t, x, x^{\prime}\right) u(t,\left.x, x^{\prime}\right) d t d x d x^{\prime}= \\
&=c \int_{\mathbb{R} \times \mathbb{R}^{d}} e^{2 i v_{0} \cdot z} u\left(t, v_{0} t+z, v_{0} t-z\right) d z d t
\end{aligned}
$$

Equivalently, $\gamma\left(t, x, x^{\prime}\right)=\delta\left(\frac{x+x^{\prime}}{2}-v_{0} t\right) e^{i v_{0} \cdot\left(x-x^{\prime}\right)}$. If $v_{0}=0$ then $\gamma$ obviously solves (13); by a Galilean shift, $\gamma$ solves (13) for arbitrary $v_{0} \in \mathbb{R}^{d}$. Therefore, the "fundamental solution" for (12) transforms into a solution of (13) under the inverse Wigner transform. We conclude that any classical state (evolving under free transport) can be represented by a distribution $\gamma\left(t, x, x^{\prime}\right)$ (evolving via a linear Schrödinger equation). Let us also point out that the inverse Wigner transform regarded as a map $f \in L_{x, v}^{2} \mapsto \gamma \in L_{x, x^{\prime}}^{2}$ is an isometric isomorphism; and, these spaces are preserved by either (12) or (13) respectively. Hence the equivalence of (12) and (13) is reflected at the $L^{2}$ level of regularity.

Remark. If $\phi(t, x)$ solves the Schrödinger equation

$$
\left(i \partial_{t}+\frac{1}{2} \Delta_{x}\right) \phi(t, x)=0
$$

then the function $\gamma\left(t, x, x^{\prime}\right)=\phi(t, x) \overline{\phi\left(t, x^{\prime}\right)}$ solves (13) and the Wigner transform $f$ solves (12) (though $f$ in this case need not be non-negative).

We now prove the equivalence of (12) and (13) at the $L^{2}$ level of regularity. (The same result holds if $f, \gamma$ are tempered distributions, and the proof is the same.)

Lemma 2.1. Suppose

$$
f \in L^{1}\left([0, T], L^{2}\left(\mathbb{R}_{x}^{d} \times \mathbb{R}_{v}^{d}\right)\right)
$$

and let

$$
\gamma \in L^{1}\left([0, T], L^{2}\left(\mathbb{R}_{x}^{d} \times \mathbb{R}_{x^{\prime}}^{d}\right)\right)
$$

denote the inverse Wigner transform of $f$. Then $f$ solves

$$
\left(\partial_{t}+v \cdot \nabla_{x}\right) f=0
$$

in in the sense of distributions, if and only if $\gamma$ solves

$$
\left(i \partial_{t}+\frac{1}{2}\left(\Delta_{x}-\Delta_{x^{\prime}}\right)\right) \gamma\left(t, x, x^{\prime}\right)=0
$$

in the sense of distributions.

Proof. Assume that

$$
\left(\partial_{t}+v \cdot \nabla_{x}\right) f=g
$$


Using the definition of the inverse Wigner transform we have

$$
\begin{aligned}
& i \partial_{t} \gamma\left(t, x, x^{\prime}\right)=\int_{\mathbb{R}^{d}} i \partial_{t} f\left(t, \frac{x+x^{\prime}}{2}, v\right) e^{i v \cdot\left(x-x^{\prime}\right)} d v \\
& \quad=\int_{\mathbb{R}^{d}} i\left(-v \cdot \nabla_{x} f+g\right)\left(t, \frac{x+x^{\prime}}{2}, v\right) e^{i v \cdot\left(x-x^{\prime}\right)} d v
\end{aligned}
$$

Let us focus on the transport term, $v \cdot \nabla_{x} f$. We have

$$
\begin{aligned}
\int_{\mathbb{R}^{d}} i & \left(-v \cdot \nabla_{x} f\right)\left(t, \frac{x+x^{\prime}}{2}, v\right) e^{i v \cdot\left(x-x^{\prime}\right)} d v \\
& =-i \int_{\mathbb{R}^{d}} v \cdot\left(\nabla_{x}+\nabla_{x^{\prime}}\right)\left[f\left(t, \frac{x+x^{\prime}}{2}, v\right)\right] e^{i v \cdot\left(x-x^{\prime}\right)} d v \\
& =-\int_{\mathbb{R}^{d}}\left(\nabla_{x}+\nabla_{x^{\prime}}\right)\left[f\left(t, \frac{x+x^{\prime}}{2}, v\right)\right] \cdot i v e^{i v \cdot\left(x-x^{\prime}\right)} d v \\
& =-\left(\nabla_{x}+\nabla_{x^{\prime}}\right) \cdot \int_{\mathbb{R}^{d}} f\left(t, \frac{x+x^{\prime}}{2}, v\right) i v e^{i v \cdot\left(x-x^{\prime}\right)} d v \\
& =-\left(\nabla_{x}+\nabla_{x^{\prime}}\right) \cdot \int_{\mathbb{R}^{d}} f\left(t, \frac{x+x^{\prime}}{2}, v\right) \frac{\nabla_{x}-\nabla_{x^{\prime}}}{2} e^{i v \cdot\left(x-x^{\prime}\right)} d v \\
& =-\frac{1}{2}\left(\nabla_{x}+\nabla_{x^{\prime}}\right) \cdot\left(\nabla_{x}-\nabla_{x^{\prime}}\right) \int_{\mathbb{R}^{d}} f\left(t, \frac{x+x^{\prime}}{2}, v\right) e^{i v \cdot\left(x-x^{\prime}\right)} d v \\
& =-\frac{1}{2}\left(\Delta_{x}-\Delta_{x^{\prime}}\right) \int_{\mathbb{R}^{d}} f\left(t, \frac{x+x^{\prime}}{2}, v\right) e^{i v \cdot\left(x-x^{\prime}\right)} d v \\
& =-\frac{1}{2}\left(\Delta_{x}-\Delta_{x^{\prime}}\right) \gamma\left(t, x, x^{\prime}\right)
\end{aligned}
$$

Therefore,

$$
\left(i \partial_{t}+\frac{1}{2}\left(\Delta_{x}-\Delta_{x^{\prime}}\right)\right) \gamma\left(t, x, x^{\prime}\right)=\int_{\mathbb{R}^{d}} g\left(t, \frac{x+x^{\prime}}{2}, v\right) e^{i v \cdot\left(x-x^{\prime}\right)} d v
$$

But $g$ vanishes identically if and only if its inverse Wigner transform vanishes identically. Therefore, $\gamma$ solves (13) if and only if $f$ solves (12).

2.3. The main result for the Boltzmann equation. It is possible to compute explicitly the equation satisfied by $\gamma$ if the Wigner transform $f$ is smooth with rapid decay and satisfies Boltzmann's equation, (11); see Corollary A.3 and Corollary A.4 in Appendix A. The result is as follows:

$$
\begin{gathered}
\left(i \partial_{t}+\frac{1}{2}\left(\Delta_{x}-\Delta_{x^{\prime}}\right)\right) \gamma(t)=B(\gamma(t), \gamma(t)) \\
B\left(\gamma_{1}, \gamma_{2}\right)=B^{+}\left(\gamma_{1}, \gamma_{2}\right)-B^{-}\left(\gamma_{1}, \gamma_{2}\right) \\
B^{-}\left(\gamma_{1}, \gamma_{2}\right)\left(x, x^{\prime}\right)=\frac{i}{2^{2 d} \pi^{d}} \int_{\mathbb{S}^{d-1}} d \omega \int_{\mathbb{R}^{d}} d z \hat{\mathbf{b}}^{\omega}\left(\frac{z}{2}\right) \times \\
\times \gamma_{1}\left(x-\frac{z}{4}, x^{\prime}+\frac{z}{4}\right) \gamma_{2}\left(\frac{x+x^{\prime}}{2}+\frac{z}{4}, \frac{x+x^{\prime}}{2}-\frac{z}{4}\right)
\end{gathered}
$$




$$
\begin{aligned}
& B^{+}\left(\gamma_{1}, \gamma_{2}\right)\left(x, x^{\prime}\right)=\frac{i}{2^{2 d} \pi^{d}} \int_{\mathbb{S}^{d-1}} d \omega \int_{\mathbb{R}^{d}} d z \hat{\mathbf{b}}^{\omega}\left(\frac{z}{2}\right) \times \\
& \quad \times \gamma_{1}\left(x-\frac{1}{2} P_{\omega}\left(x-x^{\prime}\right)-\frac{R_{\omega}(z)}{4}, x^{\prime}+\frac{1}{2} P_{\omega}\left(x-x^{\prime}\right)+\frac{R_{\omega}(z)}{4}\right) \times \\
& \quad \times \gamma_{2}\left(\frac{x+x^{\prime}}{2}+\frac{1}{2} P_{\omega}\left(x-x^{\prime}\right)+\frac{R_{\omega}(z)}{4}, \frac{x+x^{\prime}}{2}-\frac{1}{2} P_{\omega}\left(x-x^{\prime}\right)-\frac{R_{\omega}(z)}{4}\right)
\end{aligned}
$$

where we define

$$
\begin{gathered}
P_{\omega}(x)=(\omega \cdot x) \omega \\
R_{\omega}(x)=\left(\mathbb{I}-2 P_{\omega}\right)(x)
\end{gathered}
$$

and $\mathbb{I}(x)=x$. Solutions of Boltzmann's equation (in the $\gamma$ formulation) are understood using Duhamel's formula:

$$
\gamma(t)=e^{\frac{1}{2} i t \Delta_{ \pm}} \gamma(0)-i \int_{0}^{t} e^{\frac{1}{2} i\left(t-t_{1}\right) \Delta_{ \pm}} B\left(\gamma\left(t_{1}\right), \gamma\left(t_{1}\right)\right) d t_{1}
$$

Here $\Delta_{ \pm}=\Delta_{x}-\Delta_{x^{\prime}}$.

We are now ready to state our first main result.

Theorem 2.2. Suppose $A \in[0,1], \alpha \in\left(\frac{d-1}{2}, \infty\right), \beta \in(d, \infty), \kappa \in(0, \infty)$, and additionally $\frac{1}{\sigma} \in(\max (0,2 A-1), 2]$; fix any $\lambda \in(0, \infty)$. Consider the Boltzmann equation (24) with $\mathbf{b} \in L_{A}^{\infty}$. For any $\gamma_{0} \in H^{\alpha, \beta, \sigma, \kappa}$ there exists a unique solution $\gamma(t)$ of Boltzmann's equation on a small time interval $[0, T]$ such that

$$
\|\| \gamma(t)\left\|_{H^{\alpha, \beta, \sigma, \kappa-\lambda t}}\right\|_{L_{t \in[0, T]}^{\infty}}<\infty
$$

and

$$
\|\| B(\gamma(t), \gamma(t))\left\|_{H^{\alpha, \beta, \sigma, \kappa-\lambda t}}\right\|_{L_{t \in[0, T]}^{1}}<\infty
$$

both hold, and $\gamma(0)=\gamma_{0}$. Moreover, for some $r \in[0,1)$ we have the following: if $\left\|\gamma_{0}\right\|_{H^{\alpha, \beta, \sigma, \kappa}} \leq M$ then for all small enough $T$ depending only on $\alpha, \beta, \kappa, \sigma, \lambda$ and $M$, there holds:

$$
\begin{gathered}
T^{\frac{1}{2}(1-r)}\|\| \gamma(t)\left\|_{H^{\alpha, \beta, \sigma, \kappa-\lambda t}}\right\|_{L_{t \in[0, T]}^{\infty}}+\|\| B(\gamma(t), \gamma(t))\left\|_{H^{\alpha, \beta, \sigma, \kappa-\lambda t}}\right\|_{L_{t \in[0, T]}^{1}} \leq \\
\leq C(M, \alpha, \beta, \sigma, \kappa, \lambda) \times T^{\frac{1}{2}(1-r)}\left\|\gamma_{0}\right\|_{H^{\alpha, \beta, \sigma, \kappa}}
\end{gathered}
$$

If $A \in\left[0, \frac{1}{2}\right)$ then we may take $\lambda=0$ and $\kappa \in[0, \infty)$ and the same results hold, with the same restrictions on $\alpha, \beta, \sigma$.

Remark. If $A=0$ it is possible to optimize the proof of Theorem 2.2 and obtain the same result, with $\lambda=0$, for any $\kappa \in[0, \infty), \frac{1}{\sigma} \in(0,2]$, and $\alpha, \beta \in\left(\frac{d-1}{2}, \infty\right)$. We omit the details. 
2.4. The main result for the Boltzmann hierarchy. We now turn to the Boltzmann hierarchy. The Boltzmann hierarchy is an infinite sequence of coupled PDEs describing the evolution of densities $f^{(k)}\left(t, x_{1}, \ldots, x_{k}, v_{1}, \ldots, v_{k}\right)$ for $k \in \mathbb{N}=\{1,2,3, \ldots\}$. The densities $f^{(k)}$ are assumed to be symmetric with respect to interchange of particle indices. The Boltzmann hierarchy arises as an intermediate equation in the derivation of Boltzmann's equation from an underlying Hamiltonian evolution of many particles, [11,16, 18. We use the notation $X_{k}=\left(x_{1}, \ldots, x_{k}\right)$ and, for $i \leq j, X_{i: j}=\left(x_{i}, x_{i+1}, \ldots, x_{j}\right)$, and similarly for $V_{k}$ and $V_{i: j}$. For each $k \in \mathbb{N}$, the $k$ th equation of the Boltzmann hierarchy is written:

$$
\left(\partial_{t}+V_{k} \cdot \nabla_{X_{k}}\right) f^{(k)}\left(t, X_{k}, V_{k}\right)=C_{k+1} f^{(k+1)}\left(t, X_{k}, V_{k}\right)
$$

where the collision operator $C_{k+1}$ is split into gain and loss parts:

$$
C_{k+1} f^{(k+1)}=\sum_{i=1}^{k} C_{i, k+1}^{+} f^{(k+1)}-\sum_{i=1}^{k} C_{i, k+1}^{-} f^{(k+1)}
$$

The gain term is written

$$
\begin{aligned}
& C_{i, k+1}^{+} f^{(k+1)}\left(t, X_{k}, V_{k}\right)= \\
& =\int_{\mathbb{R}^{d} \times \mathbb{S}^{d-1}} d v_{k+1} d \omega \mathbf{b}\left(\left|v_{k+1}-v_{i}\right|, \omega \cdot \frac{v_{k+1}-v_{i}}{\left|v_{k+1}-v_{i}\right|}\right) \times \\
& \quad \times f^{(k+1)}\left(t, x_{1}, \ldots, x_{i}, \ldots, x_{k}, x_{i}, v_{1}, \ldots, v_{i}^{*}, \ldots, v_{k}, v_{k+1}^{*}\right)
\end{aligned}
$$

where

$$
\begin{aligned}
v_{i}^{*} & =v_{i}+P_{\omega}\left(v_{k+1}-v_{i}\right) \\
v_{k+1}^{*} & =v_{k+1}-P_{\omega}\left(v_{k+1}-v_{i}\right)
\end{aligned}
$$

Similarly for the loss term we have

$$
\begin{aligned}
& C_{i, k+1}^{-} f^{(k+1)}\left(t, X_{k}, V_{k}\right)= \\
& =\int_{\mathbb{R}^{d} \times \mathbb{S}^{d-1}} d v_{k+1} d \omega \mathbf{b}\left(\left|v_{k+1}-v_{i}\right|, \omega \cdot \frac{v_{k+1}-v_{i}}{\left|v_{k+1}-v_{i}\right|}\right) \times \\
& \quad \times f^{(k+1)}\left(t, x_{1}, \ldots, x_{i}, \ldots, x_{k}, x_{i}, v_{1}, \ldots, v_{i}, \ldots, v_{k}, v_{k+1}\right)
\end{aligned}
$$

Note carefully that the collision operators $C_{i, k+1}^{ \pm}$involve the evaluation of $f^{(k+1)}$ along the hypersurface $x_{k+1}=x_{i}$.

In exactly the same manner as for the Boltzmann equation, we define the Wigner tranform and its inverse for multiple particles:

$$
\begin{gathered}
\gamma^{(k)}\left(t, X_{k}, X_{k}^{\prime}\right)=\int_{\mathbb{R}^{d k}} f^{(k)}\left(t, \frac{X_{k}+X_{k}^{\prime}}{2}, V_{k}\right) e^{i V_{k} \cdot\left(X_{k}-X_{k}^{\prime}\right)} d V_{k} \\
f^{(k)}\left(t, X_{k}, V_{k}\right)=\frac{1}{(2 \pi)^{d k}} \int_{\mathbb{R}^{d k}} \gamma^{(k)}\left(t, X_{k}+\frac{Y_{k}}{2}, X_{k}-\frac{Y_{k}}{2}\right) e^{-i V_{k} \cdot Y_{k}} d Y_{k}
\end{gathered}
$$


The Fourier transform of $\gamma^{(k)}$ is written

$$
\begin{aligned}
\hat{\gamma}^{(k)}\left(\xi_{1}, \ldots, \xi_{k}, \xi_{1}^{\prime}, \ldots, \xi_{k}^{\prime}\right) & \\
& =\int e^{-i \sum_{i=1}^{k} x_{i} \cdot \xi_{i}} e^{-i \sum_{i=1}^{k} x_{i}^{\prime} \cdot \xi_{i}^{\prime}} \gamma^{(k)}\left(X_{k}, X_{k}^{\prime}\right) d X_{k} d X_{k}^{\prime}
\end{aligned}
$$

Let us define the weighted Sobolev spaces $H_{k}^{\alpha, \beta, \sigma, \kappa}$ for $\alpha, \beta, \kappa \geq 0$ and $\sigma>0$ :

$$
\begin{aligned}
& \left\|\gamma^{(k)}\left(X_{k}, X_{k}^{\prime}\right)\right\|_{H_{k}^{\alpha, \beta, \sigma, \kappa}}= \\
& =\| \prod_{i=1}^{k}\left\{\begin{array}{c}
\left\langle\xi_{i}+\xi_{i}^{\prime}\right\rangle^{\alpha}\left\langle\xi_{i}\right. \\
\left.\left.\quad-\xi_{i}^{\prime}\right\rangle^{\beta} e^{\kappa\left\langle\xi_{i}-\xi_{i}^{\prime}\right\rangle^{\frac{1}{\sigma}}}\right\} \times \\
\quad \times \hat{\gamma}^{(k)}\left(\xi_{1}, \ldots, \xi_{k}, \xi_{1}^{\prime}, \ldots, \xi_{k}^{\prime}\right)
\end{array} \|_{L_{\xi_{1}, \ldots, \xi_{k}, \xi_{1}^{\prime}, \ldots, \xi_{k}^{\prime}}^{2}}\right.
\end{aligned}
$$

These norms are equivalent (up to a factor like $C^{k}$ ) to the following norms for classical densities:

$$
\left\|\prod_{i=1}^{k}\left\{\left\langle 2 v_{i}\right\rangle^{\beta} e^{\kappa\left\langle 2 v_{i}\right\rangle^{\frac{1}{\sigma}}}\left(1-\Delta_{x_{i}}\right)^{\frac{\alpha}{2}}\right\} f^{(k)}\left(X_{k}, V_{k}\right)\right\|_{L_{X_{k}, V_{k}}^{2}}
$$

If $\Gamma=\left\{\gamma^{(k)}\right\}_{k \in \mathbb{N}}$ and $\xi>0$ then we further define

$$
\|\Gamma\|_{\mathcal{H}_{\xi}^{\alpha, \beta, \sigma, \kappa}}=\sum_{k \in \mathbb{N}} \xi^{k}\left\|\gamma^{(k)}\left(X_{k}, V_{k}\right)\right\|_{H_{k}^{\alpha, \beta, \sigma, \kappa}}
$$

Note that $\gamma \in H^{\alpha, \beta, \sigma, \kappa}$ if and only if $\Gamma=\left\{\gamma^{\otimes k}\right\}_{k \in \mathbb{N}} \in \mathcal{H}_{\xi}^{\alpha, \beta, \sigma, \kappa}$ for some (arbitrary) $\xi>0$.

The inverse Wigner transform of the Boltzmann hierarchy is: (see Proposition A.1 and Proposition A.2 in Appendix A

$$
\begin{gathered}
\left(i \partial_{t}+\frac{1}{2}\left(\Delta_{X_{k}}-\Delta_{X_{k}^{\prime}}\right)\right) \gamma^{(k)}\left(t, X_{k}, X_{k}^{\prime}\right)=B_{k+1} \gamma^{(k+1)}\left(t, X_{k}, X_{k}^{\prime}\right) \\
B_{k+1} \gamma^{(k+1)}=\sum_{i=1}^{k}\left(B_{i, k+1}^{+} \gamma^{(k+1)}-B_{i, k+1}^{-} \gamma^{(k+1)}\right) \\
B_{i, k+1}^{-} \gamma^{(k+1)}\left(t, X_{k}, X_{k}^{\prime}\right)= \\
=\frac{i}{2^{2 d} \pi^{d}} \int_{\mathbb{S}^{d-1}} d \omega \int_{\mathbb{R}^{d}} d z \hat{\mathbf{b}}^{\omega}\left(\frac{z}{2}\right) \times \\
\times \gamma^{(k+1)}\left(t, X_{1:(i-1)}, x_{i}-\frac{z}{4}, X_{(i+1): k}, \frac{x_{i}+x_{i}^{\prime}}{2}+\frac{z}{4},\right. \\
\left.X_{1:(i-1)}^{\prime}, x_{i}^{\prime}+\frac{z}{4}, X_{(i+1): k}^{\prime}, \frac{x_{i}+x_{i}^{\prime}}{2}-\frac{z}{4}\right)
\end{gathered}
$$




$$
\begin{aligned}
& B_{i, k+1}^{+} \gamma^{(k+1)}\left(t, X_{k}, X_{k}^{\prime}\right)= \\
& =\frac{i}{2^{2 d} \pi^{d}} \int_{\mathbb{S}^{d-1}} d \omega \int_{\mathbb{R}^{d}} d z \hat{\mathbf{b}}^{\omega}\left(\frac{z}{2}\right) \times \\
& \times \gamma^{(k+1)}\left(t, X_{1:(i-1)}, x_{i}-\frac{1}{2} P_{\omega}\left(x_{i}-x_{i}^{\prime}\right)-\frac{R_{\omega}(z)}{4}, X_{(i+1): k},\right. \\
& \quad \frac{x_{i}+x_{i}^{\prime}}{2}+\frac{1}{2} P_{\omega}\left(x_{i}-x_{i}^{\prime}\right)+\frac{R_{\omega}(z)}{4}, \\
& X_{1:(i-1)}^{\prime}, x_{i}^{\prime}+\frac{1}{2} P_{\omega}\left(x_{i}-x_{i}^{\prime}\right)+\frac{R_{\omega}(z)}{4}, X_{(i+1): k}^{\prime}, \\
& \left.\quad \frac{x_{i}+x_{i}^{\prime}}{2}-\frac{1}{2} P_{\omega}\left(x_{i}-x_{i}^{\prime}\right)-\frac{R_{\omega}(z)}{4}\right)
\end{aligned}
$$

Solutions of the Boltzmann hierarchy are understood using Duhamel's formula: for all $k \in \mathbb{N}$,

$$
\gamma^{(k)}(t)=e^{\frac{1}{2} i t \Delta_{ \pm}^{(k)}} \gamma^{(k)}(0)-i \int_{0}^{t} e^{\frac{1}{2} i\left(t-t_{1}\right) \Delta_{ \pm}^{(k)}} B_{k+1} \gamma^{(k+1)}\left(t_{1}\right) d t_{1}
$$

Here $\Delta_{ \pm}^{(k)}=\Delta_{X_{k}}-\Delta_{X_{k}^{\prime}}$. We further define $B \Gamma=\left\{B_{k+1} \gamma^{(k+1)}\right\}_{k \in \mathbb{N}^{*}}$.

We are ready to state our second main result.

Theorem 2.3. Suppose $\mathbf{b} \in L_{A}^{\infty}$ with $A \in\left[0, \frac{1}{2}\right), \alpha \in\left(\frac{d-1}{2}, \infty\right), \beta \in$ $(d, \infty), \kappa \in[0, \infty)$, and $\frac{1}{\sigma} \in(0,2]$. Assume $\Gamma_{0}=\left\{\gamma_{0}^{(k)}\right\}_{k \in \mathbb{N}} \in \mathcal{H}_{\xi_{1}}^{\alpha, \beta, \sigma, \kappa}$ where $\xi_{1} \in(0,1)$, and further assume that the functions $\gamma_{0}^{(k)}$ are symmetric under particle interchange. Then there exists $T>0$ and $0<\xi_{2}<\xi_{1}$ such that there exists a unique solution $\Gamma(t)$ of the Boltzmann hierarchy (45) for $t \in[0, T]$ with $\|\Gamma(t)\|_{L_{t \in[0, T]}^{\infty} \mathcal{H}_{\xi_{2}}^{\alpha, \beta, \sigma, \kappa}}<\infty$ and $\|B \Gamma(t)\|_{L_{t \in[0, T]}^{1} \mathcal{H}_{\xi_{2}}^{\alpha, \beta, \sigma, \kappa}}<\infty$, and $\Gamma(0)=\Gamma_{0}$. Moreover, the following estimate holds:

$$
\|\Gamma\|_{L_{t \in[0, T]}^{\infty} \mathcal{H}_{\xi_{2}}^{\alpha, \beta, \sigma, \kappa}}+\|B \Gamma\|_{L_{t \in[0, T]}^{1} \mathcal{H}_{\xi_{2}}^{\alpha, \beta, \sigma, \kappa}} \leq C\left\|\Gamma_{0}\right\|_{\mathcal{H}_{\xi_{1}}^{\alpha, \beta, \sigma, \kappa}}
$$

where $C$ depends on $T, d, \xi_{1}, \xi_{2}, \alpha, \beta, \sigma, \kappa$.

Remark. If $A=0$ it is possible to optimize the proof of Theorem 2.3 and obtain the same result for any $\kappa \in[0, \infty), \frac{1}{\sigma} \in(0,2]$, and $\alpha, \beta \in\left(\frac{d-1}{2}, \infty\right)$. We omit the details.

2.5. Interpretation of the Boltzmann hierarchy. Extending Theorem 2.3 to the full range $A \in[0,1]$ would require revising the boardgame argument as presented in [17] to be compatible with time-dependent weights, as in Theorem 2.2. Unfortunately this seems to be technically out of reach at the present time; indeed, it seems to be an interesting open question to determine whether the hard sphere Boltzmann hierarchy is in fact locally well-posed for data $\Gamma(0) \in \mathcal{H}_{\xi}^{\alpha, \beta, \sigma, \kappa}$ with a suitable choice of parameters.

Since we cannot (at present) extend our well-posedness result to the hard sphere Boltzmann hierarchy $(A=1)$, the reader will rightfully question why 
we study the Boltzmann hierarchy at all. After all, the hard sphere interaction is the only interaction with Grad cut-off that is physically relevant (and all our results assume the Grad cut-off). In particular, at present, we have nothing to offer in the context of Lanford's theorem, even at the level of the Boltzmann hierarchy. Nevertheless, the Boltzmann hierarchy always has an interpretation in the context of statistical solutions of the Boltzmann equation. (See 4 for a formal discussion of statistical solutions.) Under suitable regularity assumptions, if $\pi_{t}$ is a statistical solution of Boltzmann's equation, then

$$
f^{(k)}(t)=\int h^{\otimes k} d \pi_{t}(h)
$$

is a solution of the Boltzmann hierarchy (for any interaction, physical or not). Conversely, suppose the functions $f^{(k)}(t)$ (assumed smooth and growing at most exponentially in $k$ ), which solve the Boltzmann hierarchy, define the joint distribution of some exchangeable sequence of random variables $\left(x_{1}, v_{1}\right),\left(x_{2}, v_{2}\right), \ldots$ In that case, the Hewitt-Savage theorem guarantees the existence of a unique underlying $\pi_{t}$ which must be a statistical solution of Boltzmann's equation. [14

\section{THE KEY PROPOSITION}

The proofs of Theorems 2.2 and 2.3 will rely on the following proposition:

Proposition 3.1. Suppose $A \in[0,1], \alpha \in\left(\frac{d-1}{2}, \infty\right), \beta \in(d, \infty)$, and $\frac{1}{\sigma} \in$ $(\max (0,2 A-1), 2]$. Then for any $r \in[0,1)$ such that $\frac{r}{\sigma} \geq \max (0,2 A-1+\delta)$ for a small $\delta>0$ we have for all $\kappa_{0}>\kappa>0$, any $1 \leq i \leq k$, and any $\gamma_{0}^{(k+1)} \in H_{k+1}^{\alpha, \beta, \sigma, \kappa_{0}}$ the following estimates:

$$
\begin{array}{r}
\left\|B_{i, k+1}^{ \pm}\left[e^{\frac{1}{2} i t\left(\Delta_{X_{k+1}}-\Delta_{X_{k+1}^{\prime}}\right)} \gamma_{0}^{(k+1)}\right]\right\|_{L_{t}^{2} H_{k}^{\alpha, \beta, \sigma, \kappa}} \leq \\
\leq C(\alpha, \beta, \sigma, r)\|\mathbf{b}\|_{L_{A}^{\infty}}\left(1+\left(\kappa_{0}-\kappa\right)^{-\frac{1}{2} r}\right)\left\|\gamma_{0}^{(k+1)}\right\|_{H_{k+1}^{\alpha, \beta, \sigma, \kappa_{0}}}
\end{array}
$$

Moreover, if $A \in\left[0, \frac{1}{2}\right), \alpha \in\left(\frac{d-1}{2}, \infty\right), \beta \in(d, \infty)$, and $\frac{1}{\sigma} \in(0,2]$, then for any $\kappa_{0} \geq \kappa \geq 0$, any $1 \leq i \leq k$, and any $\gamma_{0}^{(k+1)} \in H_{k+1}^{\alpha, \beta, \sigma, \kappa_{0}}$ the following estimates hold:

$$
\begin{aligned}
& \| B_{i, k+1}^{ \pm}\left[e^{\frac{1}{2} i t\left(\Delta_{X_{k+1}}-\Delta_{X_{k+1}^{\prime}}\right)}\right.\left.\gamma_{0}^{(k+1)}\right] \|_{L_{t}^{2} H_{k}^{\alpha, \beta, \sigma, \kappa}} \leq \\
& \leq C(\alpha, \beta, \sigma)\|\mathbf{b}\|_{L_{A}^{\infty}}\left\|\gamma_{0}^{(k+1)}\right\|_{H_{k+1}^{\alpha, \beta, \sigma, \kappa_{0}}}
\end{aligned}
$$

Remark. Note that the second part of Proposition 3.1 formally follows from the first part by setting $r=0$. In fact we will only prove the first part since the second part follows after trivial changes to the proof. 
Remark. If $A=0$ it is possible to optimize the proof of Proposition 3.1 and obtain (53) for any $\kappa_{0} \geq \kappa \geq 0, \frac{1}{\sigma} \in(0,2]$, and $\alpha, \beta \in\left(\frac{d-1}{2}, \infty\right)$. We omit the details.

\section{Proof of Proposition 3.1.}

Loss Term. Consider a typical part of the loss term, e.g. $B_{1, k+1}^{-} \gamma^{(k+1)}$ :

$$
\begin{aligned}
& B_{1, k+1}^{-} \gamma^{(k+1)}\left(t, X_{k}, X_{k}^{\prime}\right)=\frac{i}{2^{2 d} \pi^{d}} \int_{\mathbb{S}^{d-1}} d \omega \int_{\mathbb{R}^{d}} d z \hat{\mathbf{b}}^{\omega}\left(\frac{z}{2}\right) \times \\
& \quad \times \gamma^{(k+1)}\left(t, x_{1}-\frac{z}{4}, X_{2: k}, \frac{x_{1}+x_{1}^{\prime}}{2}+\frac{z}{4}, x_{1}^{\prime}+\frac{z}{4}, X_{2: k}^{\prime}, \frac{x_{1}+x_{1}^{\prime}}{2}-\frac{z}{4}\right)
\end{aligned}
$$

We will fix some initial data $\gamma_{0}^{(k+1)}\left(X_{k+1}, X_{k+1}^{\prime}\right)$ and consider the following function:

$$
B_{1, k+1}^{-}\left[e^{\frac{1}{2} i t\left(\Delta_{X_{k+1}}-\Delta_{X_{k+1}^{\prime}}\right)} \gamma_{0}^{(k+1)}\right]\left(t, X_{k}, X_{k}^{\prime}\right)
$$

The spacetime Fourier transform of a function $F\left(t, X_{k}, X_{k}^{\prime}\right)$ is

$$
\begin{aligned}
& \tilde{F}\left(\tau, \xi_{1}, \ldots, \xi_{k}, \xi_{1}^{\prime}, \ldots, \xi_{k}^{\prime}\right)= \\
& =\int d t d X_{k} d X_{k}^{\prime} e^{-i t \tau} e^{-i \sum_{i=1}^{k} x_{i} \cdot \xi_{i}} e^{-i \sum_{i=1}^{k} x_{i}^{\prime} \cdot \xi_{i}^{\prime}} F\left(t, X_{k}, X_{k}^{\prime}\right)
\end{aligned}
$$

The spacetime Fourier transform of $e^{\frac{1}{2} i t\left(\Delta_{X_{k+1}}-\Delta_{X_{k+1}^{\prime}}\right)} \gamma_{0}^{(k+1)}$ is, up to a constant depending on $k$,

$$
\hat{\gamma}_{0}^{(k+1)}\left(\xi_{1}, \ldots, \xi_{k+1}, \xi_{1}^{\prime}, \ldots, \xi_{k+1}^{\prime}\right) \delta\left(\tau+\frac{1}{2} \sum_{i=1}^{k+1}\left|\xi_{i}\right|^{2}-\frac{1}{2} \sum_{i=1}^{k+1}\left|\xi_{i}^{\prime}\right|^{2}\right)
$$

We also have

$$
\begin{aligned}
& \left(B_{1, k+1}^{-}\left[e^{\frac{1}{2} i t\left(\Delta_{X_{k+1}}-\Delta_{X_{k+1}^{\prime}}\right)} \gamma_{0}^{(k+1)}\right]\right)^{\sim}\left(\tau, \xi_{1}, \ldots, \xi_{k}, \xi_{1}^{\prime}, \ldots, \xi_{k}^{\prime}\right)= \\
& =\text { cst. } \int d \eta d \eta^{\prime} \delta\left(\tau+\frac{1}{2}\left|\xi_{1}-\frac{\eta+\eta^{\prime}}{2}\right|^{2}-\frac{1}{2}\left|\xi_{1}^{\prime}-\frac{\eta+\eta^{\prime}}{2}\right|^{2}+\right. \\
& \left.+\frac{1}{2}|\eta|^{2}-\frac{1}{2}\left|\eta^{\prime}\right|^{2}+\frac{1}{2} \sum_{2 \leq i \leq k}\left(\left|\xi_{i}\right|^{2}-\left|\xi_{i}^{\prime}\right|^{2}\right)\right) \times \\
& \times \mathbf{b}\left(\frac{\left|-\xi_{1}+\xi_{1}^{\prime}+\eta-\eta^{\prime}\right|}{2}, \omega \cdot \frac{-\xi_{1}+\xi_{1}^{\prime}+\eta-\eta^{\prime}}{\left|-\xi_{1}+\xi_{1}^{\prime}+\eta-\eta^{\prime}\right|}\right) \times \\
& \times \hat{\gamma}_{0}^{(k+1)}\left(\xi_{1}-\frac{\eta+\eta^{\prime}}{2}, \xi_{2}, \ldots, \xi_{k}, \eta, \xi_{1}^{\prime}-\frac{\eta+\eta^{\prime}}{2}, \xi_{2}^{\prime}, \ldots, \xi_{k}^{\prime}, \eta^{\prime}\right)
\end{aligned}
$$


The constant is uniformly bounded in $k$. Now we simply bound the collision kernel b using $\|\mathbf{b}\|_{L_{A}^{\infty}}$ to yield:

$$
\begin{aligned}
& \left|\left(B_{1, k+1}^{-}\left[e^{\frac{1}{2} i t\left(\Delta_{X_{k+1}}-\Delta_{X_{k+1}^{\prime}}\right)} \gamma_{0}^{(k+1)}\right]\right)^{\sim}\left(\tau, \xi_{1}, \ldots, \xi_{k}, \xi_{1}^{\prime}, \ldots, \xi_{k}^{\prime}\right)\right| \lesssim \\
& \lesssim\|\mathbf{b}\|_{L_{A}^{\infty}} \int d \eta d \eta^{\prime}\left(\left\langle\xi_{1}-\xi_{1}^{\prime}\right\rangle^{A}+\left\langle\eta-\eta^{\prime}\right\rangle^{A}\right) \times \\
& \times \delta\left(\tau+\frac{1}{2}\left|\xi_{1}-\frac{\eta+\eta^{\prime}}{2}\right|^{2}-\frac{1}{2}\left|\xi_{1}^{\prime}-\frac{\eta+\eta^{\prime}}{2}\right|^{2}+\right. \\
& \left.+\frac{1}{2}|\eta|^{2}-\frac{1}{2}\left|\eta^{\prime}\right|^{2}+\frac{1}{2} \sum_{2 \leq i \leq k}\left(\left|\xi_{i}\right|^{2}-\left|\xi_{i}^{\prime}\right|^{2}\right)\right) \times \\
& \times\left|\hat{\gamma}_{0}^{(k+1)}\left(\xi_{1}-\frac{\eta+\eta^{\prime}}{2}, \xi_{2}, \ldots, \xi_{k}, \eta, \xi_{1}^{\prime}-\frac{\eta+\eta^{\prime}}{2}, \xi_{2}^{\prime}, \ldots, \xi_{k}^{\prime}, \eta^{\prime}\right)\right|
\end{aligned}
$$

We want to estimate the following integral, for suitable $\alpha, \beta, \kappa, \sigma>0$ :

$$
\begin{aligned}
I^{-}(\alpha, \beta, \kappa, \sigma)=\int d \tau d \xi_{1} \ldots & d \xi_{k} d \xi_{1}^{\prime} \ldots d \xi_{k}^{\prime} \times \\
\times & \prod_{i=1}^{k}\left\{\left\langle\xi_{i}+\xi_{i}^{\prime}\right\rangle^{2 \alpha}\left\langle\xi_{i}-\xi_{i}^{\prime}\right\rangle^{2 \beta} e^{2 \kappa\left\langle\xi_{i}-\xi_{i}^{\prime}\right\rangle^{\frac{1}{\sigma}}}\right\} \times \\
& \times\left|\left(B_{1, k+1}^{-}\left[e^{\frac{1}{2} i t\left(\Delta_{X_{k+1}}-\Delta_{X_{k+1}^{\prime}}\right)} \gamma_{0}^{(k+1)}\right]\right)^{\sim}\right|^{2}
\end{aligned}
$$


To start, observe that

$$
\begin{aligned}
& I^{-}(\alpha, \beta, \kappa, \sigma) \lesssim\|\mathbf{b}\|_{L_{A}^{\infty}}^{2} \int d \tau d \xi_{1} \ldots d \xi_{k} d \xi_{1}^{\prime} \ldots d \xi_{k}^{\prime} d \eta_{1} d \eta_{1}^{\prime} d \eta_{2} d \eta_{2}^{\prime} \times \\
& \times \prod_{i=1}^{k}\left\{\left\langle\xi_{i}+\xi_{i}^{\prime}\right\rangle^{2 \alpha}\left\langle\xi_{i}-\xi_{i}^{\prime}\right\rangle^{2 \beta} e^{2 \kappa\left\langle\xi_{i}-\xi_{i}^{\prime}\right\rangle^{\frac{1}{\sigma}}}\right\} \times \\
& \times\left(\left\langle\xi_{1}-\xi_{1}^{\prime}\right\rangle^{A}+\left\langle\eta_{1}-\eta_{1}^{\prime}\right\rangle^{A}\right)\left(\left\langle\xi_{1}-\xi_{1}^{\prime}\right\rangle^{A}+\left\langle\eta_{2}-\eta_{2}^{\prime}\right\rangle^{A}\right) \times \\
& \times \delta\left(\tau+\frac{1}{2}\left|\xi_{1}-\frac{\eta_{1}+\eta_{1}^{\prime}}{2}\right|^{2}-\frac{1}{2}\left|\xi_{1}^{\prime}-\frac{\eta_{1}+\eta_{1}^{\prime}}{2}\right|^{2}+\right. \\
& \left.+\frac{1}{2}\left|\eta_{1}\right|^{2}-\frac{1}{2}\left|\eta_{1}^{\prime}\right|^{2}+\frac{1}{2} \sum_{2 \leq i \leq k}\left(\left|\xi_{i}\right|^{2}-\left|\xi_{i}^{\prime}\right|^{2}\right)\right) \times \\
& \times \delta\left(\tau+\frac{1}{2}\left|\xi_{1}-\frac{\eta_{2}+\eta_{2}^{\prime}}{2}\right|^{2}-\frac{1}{2}\left|\xi_{1}^{\prime}-\frac{\eta_{2}+\eta_{2}^{\prime}}{2}\right|^{2}+\right. \\
& \left.+\frac{1}{2}\left|\eta_{2}\right|^{2}-\frac{1}{2}\left|\eta_{2}^{\prime}\right|^{2}+\frac{1}{2} \sum_{2 \leq i \leq k}\left(\left|\xi_{i}\right|^{2}-\left|\xi_{i}^{\prime}\right|^{2}\right)\right) \times \\
& \times\left|\hat{\gamma}_{0}^{(k+1)}\left(\xi_{1}-\frac{\eta_{1}+\eta_{1}^{\prime}}{2}, \xi_{2}, \ldots, \xi_{k}, \eta_{1}, \xi_{1}^{\prime}-\frac{\eta_{1}+\eta_{1}^{\prime}}{2}, \xi_{2}^{\prime}, \ldots, \xi_{k}^{\prime}, \eta_{1}^{\prime}\right)\right| \times \\
& \times\left|\hat{\gamma}_{0}^{(k+1)}\left(\xi_{1}-\frac{\eta_{2}+\eta_{2}^{\prime}}{2}, \xi_{2}, \ldots, \xi_{k}, \eta_{2}, \xi_{1}^{\prime}-\frac{\eta_{2}+\eta_{2}^{\prime}}{2}, \xi_{2}^{\prime}, \ldots, \xi_{k}^{\prime}, \eta_{2}^{\prime}\right)\right|
\end{aligned}
$$

Let $\kappa_{0}>\kappa$, then multiply and divide the integrand by the following factor:

$$
\prod_{j=1}^{2}\left\{\begin{array}{c}
\left\langle\xi_{1}+\xi_{1}^{\prime}-\eta_{j}-\eta_{j}^{\prime}\right\rangle^{\alpha}\left\langle\xi_{1}-\xi_{1}^{\prime}\right\rangle^{\beta} e^{\kappa_{0}\left\langle\xi_{1}-\xi_{1}^{\prime}\right\rangle^{\frac{1}{\sigma}} \times} \\
\times\left\langle\eta_{j}+\eta_{j}^{\prime}\right\rangle^{\alpha}\left\langle\eta_{j}-\eta_{j}^{\prime}\right\rangle^{\beta} e^{\kappa_{0}\left\langle\eta_{j}-\eta_{j}^{\prime}\right\rangle^{\frac{1}{\sigma}}}
\end{array}\right\}
$$

Then group terms together and apply Cauchy-Schwarz pointwise under the integral sign. We obtain two different terms that are equal due to symmetry 
under re-labeling coordinates; hence,

$$
\begin{aligned}
& I^{-} \lesssim\|\mathbf{b}\|_{L_{A}^{\infty}}^{2} \int d \tau d \xi_{1} \ldots d \xi_{k} d \xi_{1}^{\prime} \ldots d \xi_{k}^{\prime} d \eta_{1} d \eta_{1}^{\prime} d \eta_{2} d \eta_{2}^{\prime} \times \\
& \times \frac{\left\langle\xi_{1}+\xi_{1}^{\prime}\right\rangle^{2 \alpha}\left\langle\xi_{1}-\xi_{1}^{\prime}\right\rangle^{2 \beta}\left(\left\langle\xi_{1}-\xi_{1}^{\prime}\right\rangle^{2 A}+\left\langle\eta_{1}-\eta_{1}^{\prime}\right\rangle^{2 A}\right)}{\left\langle\xi_{1}+\xi_{1}^{\prime}-\eta_{1}-\eta_{1}^{\prime}\right\rangle^{2 \alpha}\left\langle\xi_{1}-\xi_{1}^{\prime}\right\rangle^{2 \beta}\left\langle\eta_{1}+\eta_{1}^{\prime}\right\rangle^{2 \alpha}\left\langle\eta_{1}-\eta_{1}^{\prime}\right\rangle^{2 \beta}} \times \\
& \times \frac{e^{2 \kappa\left\langle\xi_{1}-\xi_{1}^{\prime}\right\rangle^{\frac{1}{\sigma}}}}{e^{2 \kappa_{0}\left\langle\xi_{1}-\xi_{1}^{\prime}\right\rangle^{\frac{1}{\sigma}}} e^{2 \kappa_{0}\left\langle\eta_{1}-\eta_{1}^{\prime}\right\rangle^{\frac{1}{\sigma}}} \times} \\
& \times \delta\left(\tau+\frac{1}{2}\left|\xi_{1}-\frac{\eta_{1}+\eta_{1}^{\prime}}{2}\right|^{2}-\frac{1}{2}\left|\xi_{1}^{\prime}-\frac{\eta_{1}+\eta_{1}^{\prime}}{2}\right|^{2}+\right. \\
& \left.+\frac{1}{2}\left|\eta_{1}\right|^{2}-\frac{1}{2}\left|\eta_{1}^{\prime}\right|^{2}+\frac{1}{2} \sum_{2 \leq i \leq k}\left(\left|\xi_{i}\right|^{2}-\left|\xi_{i}^{\prime}\right|^{2}\right)\right) \times \\
& \times \delta\left(\tau+\frac{1}{2}\left|\xi_{1}-\frac{\eta_{2}+\eta_{2}^{\prime}}{2}\right|^{2}-\frac{1}{2}\left|\xi_{1}^{\prime}-\frac{\eta_{2}+\eta_{2}^{\prime}}{2}\right|^{2}+\right. \\
& \left.+\frac{1}{2}\left|\eta_{2}\right|^{2}-\frac{1}{2}\left|\eta_{2}^{\prime}\right|^{2}+\frac{1}{2} \sum_{2 \leq i \leq k}\left(\left|\xi_{i}\right|^{2}-\left|\xi_{i}^{\prime}\right|^{2}\right)\right) \times \\
& \times\left\langle\xi_{1}+\xi_{1}^{\prime}-\eta_{2}-\eta_{2}^{\prime}\right\rangle^{2 \alpha}\left\langle\xi_{1}-\xi_{1}^{\prime}\right\rangle^{2 \beta} e^{2 \kappa_{0}\left\langle\xi_{1}-\xi_{1}^{\prime}\right\rangle^{\frac{1}{\sigma}} \times} \\
& \times\left\langle\eta_{2}+\eta_{2}^{\prime}\right\rangle^{2 \alpha}\left\langle\eta_{2}-\eta_{2}^{\prime}\right\rangle^{2 \beta} e^{2 \kappa_{0}\left\langle\eta_{2}-\eta_{2}^{\prime}\right\rangle^{\frac{1}{\sigma}} \times} \\
& \times \prod_{2 \leq i \leq k}\left\{\left\langle\xi_{i}+\xi_{i}^{\prime}\right\rangle^{2 \alpha}\left\langle\xi_{i}-\xi_{i}^{\prime}\right\rangle^{2 \beta} e^{2 \kappa_{0}\left\langle\xi_{i}-\xi_{i}^{\prime}\right\rangle^{\frac{1}{\sigma}}}\right\} \times \\
& \times\left|\hat{\gamma}_{0}^{(k+1)}\left(\xi_{1}-\frac{\eta_{2}+\eta_{2}^{\prime}}{2}, \xi_{2}, \ldots, \xi_{k}, \eta_{2}, \xi_{1}^{\prime}-\frac{\eta_{2}+\eta_{2}^{\prime}}{2}, \xi_{2}^{\prime}, \ldots, \xi_{k}^{\prime}, \eta_{2}^{\prime}\right)\right|^{2}
\end{aligned}
$$

The integral completely factorizes in the following way:

$$
\begin{aligned}
I^{-} & \leq \int d \tau d \xi_{1} \ldots d \xi_{k} d \xi_{1}^{\prime} \ldots d \xi_{k}^{\prime}\left(\int d \eta_{1} d \eta_{1}^{\prime} \ldots\right)\left(\int d \eta_{2} d \eta_{2}^{\prime} \ldots\right) \\
& \leq\left(\sup _{\tau, \xi_{i}, \xi_{i}^{\prime}} \int d \eta_{1} d \eta_{1}^{\prime} \ldots\right) \times \int d \tau d \xi_{1} \ldots d \xi_{k} d \xi_{1}^{\prime} \ldots d \xi_{k}^{\prime}\left(\int d \eta_{2} d \eta_{2}^{\prime} \ldots\right)
\end{aligned}
$$


Finally we are able to conclude that if the following integral,

$$
\begin{aligned}
& \int d \eta d \eta^{\prime} \delta\left(\tau+\frac{1}{2}\left|\xi_{1}-\frac{\eta+\eta^{\prime}}{2}\right|^{2}-\frac{1}{2}\left|\xi_{1}^{\prime}-\frac{\eta+\eta^{\prime}}{2}\right|^{2}+\right. \\
&\left.+\frac{1}{2}|\eta|^{2}-\frac{1}{2}\left|\eta^{\prime}\right|^{2}+\frac{1}{2} \sum_{2 \leq i \leq k}\left(\left|\xi_{i}\right|^{2}-\left|\xi_{i}^{\prime}\right|^{2}\right)\right) \times \\
& \times \frac{\left\langle\xi_{1}+\xi_{1}^{\prime}\right\rangle^{2 \alpha}\left(\left\langle\xi_{1}-\xi_{1}^{\prime}\right\rangle^{2 A}+\left\langle\eta-\eta^{\prime}\right\rangle^{2 A}\right)}{\left\langle\xi_{1}+\xi_{1}^{\prime}-\eta-\eta^{\prime}\right\rangle^{2 \alpha}\left\langle\eta+\eta^{\prime}\right\rangle^{2 \alpha}\left\langle\eta-\eta^{\prime}\right\rangle^{2 \beta}} e^{-2\left(\kappa_{0}-\kappa\right)\left\langle\xi_{1}-\xi_{1}^{\prime}\right\rangle^{\frac{1}{\sigma}}}
\end{aligned}
$$

is bounded uniformly with respect to $\tau, \xi_{1}, \ldots, \xi_{k}, \xi_{1}^{\prime}, \ldots, \xi_{k}^{\prime}$, then the following estimate holds:

$$
\begin{aligned}
& \| B_{1, k+1}^{-}\left[e^{\frac{1}{2} i t\left(\Delta_{X_{k+1}}-\Delta_{X_{k+1}^{\prime}}\right)} \gamma_{0}^{(k+1)}\right] \|_{L_{t}^{2} H_{k}^{\alpha, \beta, \sigma, \kappa}} \leq \\
& \leq C\left(\alpha, \beta, \sigma, \kappa, \kappa_{0}\right)\|\mathbf{b}\|_{L_{A}^{\infty}}\left\|\gamma_{0}^{(k+1)}\right\|_{H_{k+1}^{\alpha, \beta, \sigma, \kappa}}
\end{aligned}
$$

Let us make the change of variables $w=\frac{\eta+\eta^{\prime}}{2}, z=\frac{\eta-\eta^{\prime}}{2}$ in (62); then, up to a constant, the integral becomes:

$$
\begin{array}{r}
\int d w d z \delta\left(\tau+\frac{1}{2}\left|\xi_{1}-w\right|^{2}-\frac{1}{2}\left|\xi_{1}^{\prime}-w\right|^{2}+\frac{1}{2}|w+z|^{2}-\frac{1}{2}|w-z|^{2}+\right. \\
\left.+\frac{1}{2} \sum_{2 \leq i \leq k}\left(\left|\xi_{i}\right|^{2}-\left|\xi_{i}^{\prime}\right|^{2}\right)\right) \times \\
\times \frac{\left\langle\xi_{1}+\xi_{1}^{\prime}\right\rangle^{2 \alpha}\left(\left\langle\xi_{1}-\xi_{1}^{\prime}\right\rangle^{2 A}+\langle 2 z\rangle^{2 A}\right)}{\left\langle\xi_{1}+\xi_{1}^{\prime}-2 w\right\rangle^{2 \alpha} e^{2\left(\kappa_{0}-\kappa\right)\left\langle\xi_{1}-\xi_{1}^{\prime}\right\rangle^{\frac{1}{\sigma}}}\langle 2 w\rangle^{2 \alpha}\langle 2 z\rangle^{2 \beta}}
\end{array}
$$

This is the same as:

$$
\begin{aligned}
K=\int d w d z \delta & \left(\tau+\frac{1}{2} \sum_{i=1}^{k}\left(\left|\xi_{i}\right|^{2}-\left|\xi_{i}^{\prime}\right|^{2}\right)-\left(\xi_{1}-\xi_{1}^{\prime}-2 z\right) \cdot w\right) \times \\
& \times \frac{\left\langle\xi_{1}+\xi_{1}^{\prime}\right\rangle^{2 \alpha}\left(\left\langle\xi_{1}-\xi_{1}^{\prime}\right\rangle^{2 A}+\langle 2 z\rangle^{2 A}\right)}{\left\langle\xi_{1}+\xi_{1}^{\prime}-2 w\right\rangle^{2 \alpha} e^{2\left(\kappa_{0}-\kappa\right)\left\langle\xi_{1}-\xi_{1}^{\prime}\right\rangle^{\frac{1}{\sigma}}\langle 2 w\rangle^{2 \alpha}\langle 2 z\rangle^{2 \beta}}}
\end{aligned}
$$

Hence, one way to parametrize the integral is to let $z \in \mathbb{R}^{d}$ be arbitrary and let $w$ range over a codimension one hyperplane in $\mathbb{R}^{d}$; the hyperplane 
is determined by $\tau, \xi, \xi^{\prime}, z$. We have:

$K \leq \int_{\mathbb{R}^{d}} d z \frac{\left\langle\xi_{1}-\xi_{1}^{\prime}\right\rangle^{2 A}+\langle 2 z\rangle^{2 A}}{\left|\xi_{1}-\xi_{1}^{\prime}-2 z\right| e^{2\left(\kappa_{0}-\kappa\right)\left\langle\xi_{1}-\xi_{1}^{\prime}\right\rangle^{\frac{1}{\sigma}}}\langle 2 z\rangle^{2 \beta}} \int_{P} \frac{d S(w)\left\langle\xi_{1}+\xi_{1}^{\prime}\right\rangle^{2 \alpha}}{\left\langle\xi_{1}+\xi_{1}^{\prime}-2 w\right\rangle^{2 \alpha}\langle 2 w\rangle^{2 \alpha}}$

where $d S(w)$ is the induced surface measure on a hyperplane $P \subset \mathbb{R}^{d}$, given explicitly by

$$
P=\left\{w \in \mathbb{R}^{d} \mid \tau+\frac{1}{2} \sum_{i=1}^{k}\left(\left|\xi_{i}\right|^{2}-\left|\xi_{i}^{\prime}\right|^{2}\right)-\left(\xi_{1}-\xi_{1}^{\prime}-2 z\right) \cdot w=0\right\}
$$

In order to show the uniform boundedness of $K$ with respect to $\tau, \xi_{1}, \ldots, \xi_{k}$, $\xi_{1}^{\prime}, \ldots, \xi_{k}^{\prime}$, it suffices to prove the uniform boundedness of the following three quantities with respect to $W \in \mathbb{R}^{d}$ :

$$
\begin{gathered}
I_{1}=\sup _{P \subset \mathbb{R}^{d}: \operatorname{dim} P=d-1} \int_{P} d S(w) \frac{\langle W\rangle^{2 \alpha}}{\langle W-w\rangle^{2 \alpha}\langle w\rangle^{2 \alpha}} \\
I_{2}=\int_{\mathbb{R}^{d}} d z \frac{1}{|W-z|\langle z\rangle^{2 \beta-2 A}} \\
I_{3}=\int_{\mathbb{R}^{d}} d z \frac{\langle W\rangle^{2 A}}{e^{2\left(\kappa_{0}-\kappa\right)\langle W\rangle^{\frac{1}{\sigma}}}|W-z|\langle z\rangle^{2 \beta}}
\end{gathered}
$$

Note that in the expression for $I_{1}, P$ is an arbitrary hyperplane of codimension one in $\mathbb{R}^{d}$.

We begin with $I_{3}$; clearly the integral over the set $|z-W|<1$ is uniformly bounded in $W$ if $\beta \geq A$. Therefore it suffices to bound the following integral:

$$
I_{3}^{\prime}=\int_{\mathbb{R}^{d}} d z \frac{\langle W\rangle^{2 A}}{e^{2\left(\kappa_{0}-\kappa\right)\langle W\rangle^{\frac{1}{\sigma}}}\langle W-z\rangle\langle z\rangle^{2 \beta}}
$$

We have the following inequality:

$$
\begin{aligned}
e^{2\left(\kappa-\kappa_{0}\right)\langle W\rangle^{\frac{1}{\sigma}}} & \geq 1+2\left(\kappa_{0}-\kappa\right)\langle W\rangle^{\frac{1}{\sigma}} \\
& \gtrsim\left(\kappa_{0}-\kappa\right)^{r}\langle W\rangle^{\frac{r}{\sigma}}
\end{aligned}
$$

where $0 \leq r \leq 1$. Since $\frac{1}{\sigma}>\max (0,(2 A-1))$, we can always find an $r \in[0,1)$ such that $\frac{r}{\sigma} \geq \max (0,(2 A-1))$. For any such value of $r$, we have:

$$
I_{3}^{\prime} \lesssim\left(\kappa_{0}-\kappa\right)^{-r} \int_{\mathbb{R}^{d}} d z \frac{\langle W\rangle}{\langle W-z\rangle\langle z\rangle^{2 \beta}}
$$

Splitting the integral into the regions $|z|<\frac{1}{2}|W|,|z|>2|W|$, and $\frac{1}{2}|W| \leq$ $|z| \leq 2|W|$, we are able to show that $I_{3} \lesssim\left(\kappa_{0}-\kappa\right)^{-r}$ uniformly in $W$ as long as $\beta>\frac{d+1}{2}, \frac{1}{\sigma}>\max (0,(2 A-1))$, and $r \in[0,1)$ is such that $\frac{r}{\sigma} \geq \max (0,(2 A-1))$. 
Let us turn to $I_{2}$; clearly, the integral over the set $|z-W|<1$ is uniformly bounded in $W$ if $\beta \geq A$. Therefore, it suffices to bound the following integral uniformly in $W$ :

$$
I_{2}^{\prime}=\int_{\mathbb{R}^{d}} d z \frac{1}{\langle W-z\rangle\langle z\rangle^{2 \beta-2 A}}
$$

For any $A \in[0,1]$, this integral is automatically bounded, uniformly in $W$, if $\beta>\frac{d+2}{2}$.

Finally we turn to $I_{1}$ :

$$
I_{1}=\sup _{P \subset \mathbb{R}^{d}: \operatorname{dim} P=d-1} \int_{P} d S(w) \frac{\langle W\rangle^{2 \alpha}}{\langle W-w\rangle^{2 \alpha}\langle w\rangle^{2 \alpha}}
$$

We consider separately the regions $|w|<\frac{1}{2}|W|,|w|>2|W|$, and $\frac{1}{2}|W| \leq$ $|w| \leq 2|W|$; we find that the integral is uniformly bounded in $W$ and $P$ as long as $\alpha>\frac{d-1}{2}$.

To summarize, as long as $\alpha>\frac{d-1}{2}, \beta>d, \frac{1}{\sigma}>\max (0,(2 A-1))$, and $r \in[0,1)$ is chosen such that $\frac{r}{\sigma} \geq \max (0,(2 A-1))$, then for all $\kappa_{0}>\kappa>0$ :

$$
\begin{array}{r}
\left\|B_{i, k+1}^{-}\left[e^{\frac{1}{2} i t\left(\Delta_{X_{k+1}}-\Delta_{X_{k+1}^{\prime}}\right)} \gamma_{0}^{(k+1)}\right]\right\|_{L_{t}^{2} H_{k}^{\alpha, \beta, \sigma, \kappa}} \leq \\
\quad \leq C(\alpha, \beta, \sigma, r)\|\mathbf{b}\|_{L_{A}^{\infty}}\left(1+\left(\kappa_{0}-\kappa\right)^{-\frac{1}{2} r}\right)\left\|\gamma_{0}^{(k+1)}\right\|_{H_{k+1}^{\alpha, \beta, \sigma, \kappa_{0}}}
\end{array}
$$

Gain Term. Consider a typical part of the gain term, e.g. $B_{1, k+1}^{+} \gamma^{(k+1)}$ :

$$
\begin{aligned}
& B_{1, k+1}^{+} \gamma^{(k+1)}\left(t, X_{k}, X_{k}^{\prime}\right)= \\
& =\frac{i}{2^{2 d} \pi^{d}} \int_{\mathbb{S}^{d-1}} d \omega \int_{\mathbb{R}^{d}} d z \hat{\mathbf{b}}^{\omega}\left(\frac{z}{2}\right) \times \\
& \times \gamma^{(k+1)}\left(t, x_{1}-\frac{1}{2} P_{\omega}\left(x_{1}-x_{1}^{\prime}\right)-\frac{R_{\omega}(z)}{4}, X_{2: k},\right. \\
& \quad \frac{x_{1}+x_{1}^{\prime}}{2}+\frac{1}{2} P_{\omega}\left(x_{1}-x_{1}^{\prime}\right)+\frac{R_{\omega}(z)}{4}, \\
& x_{1}^{\prime}+\frac{1}{2} P_{\omega}\left(x_{1}-x_{1}^{\prime}\right)+\frac{R_{\omega}(z)}{4}, X_{2: k}^{\prime}, \\
& \left.\frac{x_{1}+x_{1}^{\prime}}{2}-\frac{1}{2} P_{\omega}\left(x_{1}-x_{1}^{\prime}\right)-\frac{R_{\omega}(z)}{4}\right)
\end{aligned}
$$

The spacetime Fourier transform of the function

$$
B_{1, k+1}^{+}\left[e^{\frac{1}{2} i t\left(\Delta_{X_{k+1}}-\Delta_{X_{k+1}^{\prime}}\right)} \gamma_{0}^{(k+1)}\right]\left(t, X_{k}, X_{k}^{\prime}\right)
$$


is the following, up to a constant:

$$
\begin{aligned}
& \int_{\mathbb{S}^{d-1}} d \omega \int d \eta_{1} d \eta_{1}^{\prime} d \eta_{2} d \eta_{2}^{\prime} \times \\
& \times \delta\left(\tau+\frac{1}{2}\left|\eta_{1}\right|^{2}-\frac{1}{2}\left|\eta_{1}^{\prime}\right|^{2}+\frac{1}{2}\left|\eta_{2}\right|^{2}-\frac{1}{2}\left|\eta_{2}^{\prime}\right|^{2}+\frac{1}{2} \sum_{2 \leq i \leq k}\left(\left|\xi_{i}\right|^{2}-\left|\xi_{i}^{\prime}\right|^{2}\right)\right) \times \\
& \times \delta\left(-\xi_{1}+\eta_{1}+\frac{\eta_{2}+\eta_{2}^{\prime}}{2}-\frac{1}{2} P_{\omega}\left(\eta_{1}-\eta_{1}^{\prime}\right)+\frac{1}{2} P_{\omega}\left(\eta_{2}-\eta_{2}^{\prime}\right)\right) \times \\
& \times \delta\left(-\xi_{1}^{\prime}+\eta_{1}^{\prime}+\frac{\eta_{2}+\eta_{2}^{\prime}}{2}+\frac{1}{2} P_{\omega}\left(\eta_{1}-\eta_{1}^{\prime}\right)-\frac{1}{2} P_{\omega} \cdot\left(\eta_{2}-\eta_{2}^{\prime}\right)\right) \times \\
& \times \mathbf{b}\left(\frac{\left|-\eta_{1}+\eta_{1}^{\prime}+\eta_{2}-\eta_{2}^{\prime}\right|}{2}, \omega \cdot \frac{R_{\omega}\left(-\eta_{1}+\eta_{1}^{\prime}+\eta_{2}-\eta_{2}^{\prime}\right)}{\left|-\eta_{1}+\eta_{1}^{\prime}+\eta_{2}-\eta_{2}^{\prime}\right|}\right) \times \\
& \times \hat{\gamma}_{0}^{(k+1)}\left(\eta_{1}, \xi_{2}, \ldots, \xi_{k}, \eta_{2}, \eta_{1}^{\prime}, \xi_{2}^{\prime}, \ldots, \xi_{k}^{\prime}, \eta_{2}^{\prime}\right)
\end{aligned}
$$

This is bounded by $\|\mathbf{b}\|_{L_{A}^{\infty}}$ times the following integral:

$$
\begin{aligned}
& \int_{\mathbb{S}^{d-1}} d \omega \int d \eta_{1} d \eta_{1}^{\prime} d \eta_{2} d \eta_{2}^{\prime}\left(\left\langle\eta_{1}-\eta_{1}^{\prime}\right\rangle^{A}+\left\langle\eta_{2}-\eta_{2}^{\prime}\right\rangle^{A}\right) \times \\
& \times \delta\left(\tau+\frac{1}{2}\left|\eta_{1}\right|^{2}-\frac{1}{2}\left|\eta_{1}^{\prime}\right|^{2}+\frac{1}{2}\left|\eta_{2}\right|^{2}-\frac{1}{2}\left|\eta_{2}^{\prime}\right|^{2}+\frac{1}{2} \sum_{2 \leq i \leq k}\left(\left|\xi_{i}\right|^{2}-\left|\xi_{i}^{\prime}\right|^{2}\right)\right) \times \\
& \times \delta\left(-\xi_{1}+\eta_{1}+\frac{\eta_{2}+\eta_{2}^{\prime}}{2}-\frac{1}{2} P_{\omega}\left(\eta_{1}-\eta_{1}^{\prime}\right)+\frac{1}{2} P_{\omega}\left(\eta_{2}-\eta_{2}^{\prime}\right)\right) \times \\
& \times \delta\left(-\xi_{1}^{\prime}+\eta_{1}^{\prime}+\frac{\eta_{2}+\eta_{2}^{\prime}}{2}+\frac{1}{2} P_{\omega}\left(\eta_{1}-\eta_{1}^{\prime}\right)-\frac{1}{2} P_{\omega}\left(\eta_{2}-\eta_{2}^{\prime}\right)\right) \times \\
& \times\left|\hat{\gamma}_{0}^{(k+1)}\left(\eta_{1}, \xi_{2}, \ldots, \xi_{k}, \eta_{2}, \eta_{1}^{\prime}, \xi_{2}^{\prime}, \ldots, \xi_{k}^{\prime}, \eta_{2}^{\prime}\right)\right|
\end{aligned}
$$


Introduce the change of variables $w_{1}=\frac{\eta_{1}+\eta_{1}^{\prime}}{2}, z_{1}=\frac{\eta_{1}-\eta_{1}^{\prime}}{2}, w_{2}=\frac{\eta_{2}+\eta_{2}^{\prime}}{2}$, $z_{2}=\frac{\eta_{2}-\eta_{2}^{\prime}}{2}$. Then (80) becomes

$$
\begin{aligned}
& \int_{\mathbb{S}^{d-1}} d \omega \int d w_{1} d z_{1} d w_{2} d z_{2}\left(\left\langle 2 z_{1}\right\rangle^{A}+\left\langle 2 z_{2}\right\rangle^{A}\right) \times \\
& \times \delta\left(\tau+\frac{1}{2}\left|w_{1}+z_{1}\right|^{2}-\frac{1}{2}\left|w_{1}-z_{1}\right|^{2}+\frac{1}{2}\left|w_{2}+z_{2}\right|^{2}-\frac{1}{2}\left|w_{2}-z_{2}\right|^{2}+\right. \\
& \left.\quad+\frac{1}{2} \sum_{2 \leq i \leq k}\left(\left|\xi_{i}\right|^{2}-\left|\xi_{i}^{\prime}\right|^{2}\right)\right) \times \\
& \times \delta\left(-\xi_{1}+w_{1}+z_{1}+w_{2}-P_{\omega}\left(z_{1}-z_{2}\right)\right) \times \\
& \times \delta\left(-\xi_{1}^{\prime}+w_{1}-z_{1}+w_{2}+P_{\omega}\left(z_{1}-z_{2}\right)\right) \times \\
& \times\left|\hat{\gamma}_{0}^{(k+1)}\left(w_{1}+z_{1}, \xi_{2}, \ldots, \xi_{k}, w_{2}+z_{2}, w_{1}-z_{1}, \xi_{2}^{\prime}, \ldots, \xi_{k}^{\prime}, w_{2}-z_{2}\right)\right|
\end{aligned}
$$

Introduce yet another change of variables $r_{1}=\frac{w_{1}+w_{2}}{2}, s_{1}=\frac{w_{1}-w_{2}}{2}, r_{2}=$ $\frac{z_{1}+z_{2}}{2}, s_{2}=\frac{z_{1}-z_{2}}{2}$. Then (181) becomes

$$
\begin{aligned}
& \int_{\mathbb{S}^{d-1}} d \omega \int d r_{1} d s_{1} d r_{2} d s_{2}\left(\left\langle 2\left(r_{2}+s_{2}\right)\right\rangle^{A}+\left\langle 2\left(r_{2}-s_{2}\right)\right\rangle^{A}\right) \times \\
& \times \delta\left(\tau+\frac{1}{2}\left|r_{1}+s_{1}+r_{2}+s_{2}\right|^{2}-\frac{1}{2}\left|r_{1}+s_{1}-r_{2}-s_{2}\right|^{2}+\right. \\
& \quad+\frac{1}{2}\left|r_{1}-s_{1}+r_{2}-s_{2}\right|^{2}-\frac{1}{2}\left|r_{1}-s_{1}-r_{2}+s_{2}\right|^{2}+ \\
& \left.\quad+\frac{1}{2} \sum_{2 \leq i \leq k}\left(\left|\xi_{i}\right|^{2}-\left|\xi_{i}^{\prime}\right|^{2}\right)\right) \times \\
& \times \delta\left(-\xi_{1}+2 r_{1}+r_{2}+R_{\omega}\left(s_{2}\right)\right) \times \\
& \times \delta\left(-\xi_{1}^{\prime}+2 r_{1}-r_{2}-R_{\omega}\left(s_{2}\right)\right) \times \\
& \times \mid \hat{\gamma}_{0}^{(k+1)}\left(r_{1}+s_{1}+r_{2}+s_{2}, \xi_{2}, \ldots, \xi_{k}, r_{1}-s_{1}+r_{2}-s_{2},\right. \\
& \left.r_{1}+s_{1}-r_{2}-s_{2}, \xi_{2}^{\prime}, \ldots, \xi_{k}^{\prime}, r_{1}-s_{1}-r_{2}+s_{2}\right) \mid
\end{aligned}
$$


Replace $r_{1}$ with $\frac{r_{1}}{2}$ throughout:

$$
\begin{aligned}
& \int_{\mathbb{S}^{d-1}} d \omega \int d r_{1} d s_{1} d r_{2} d s_{2}\left(\left\langle 2\left(r_{2}+s_{2}\right)\right\rangle^{A}+\left\langle 2\left(r_{2}-s_{2}\right)\right\rangle^{A}\right) \times \\
& \times \delta\left(\tau+\frac{1}{2}\left|\frac{r_{1}}{2}+s_{1}+r_{2}+s_{2}\right|^{2}-\frac{1}{2}\left|\frac{r_{1}}{2}+s_{1}-r_{2}-s_{2}\right|^{2}+\right. \\
& \quad+\frac{1}{2}\left|\frac{r_{1}}{2}-s_{1}+r_{2}-s_{2}\right|^{2}-\frac{1}{2}\left|\frac{r_{1}}{2}-s_{1}-r_{2}+s_{2}\right|^{2}+ \\
& \left.\left.\times \delta\left(-\xi_{1}+r_{1}+r_{2}+R_{\omega}\left(s_{2}\right)\right) \times\left|\xi_{i}^{\prime}\right|^{2}\right)\right) \times \\
& \times \delta\left(-\xi_{1}^{\prime}+r_{1}-r_{2}-R_{\omega}\left(s_{2}\right)\right) \times \\
& \times \mid \hat{\gamma}_{0}^{(k+1)}\left(\frac{r_{1}}{2}+s_{1}+r_{2}+s_{2}, \xi_{2}, \ldots, \xi_{k}, \frac{r_{1}}{2}-s_{1}+r_{2}-s_{2},\right. \\
& \left.\frac{r_{1}}{2}+s_{1}-r_{2}-s_{2}, \xi_{2}^{\prime}, \ldots, \xi_{k}^{\prime}, \frac{r_{1}}{2}-s_{1}-r_{2}+s_{2}\right) \mid
\end{aligned}
$$

Finally perform the change of variables $\zeta_{1}=r_{1}+r_{2}, \zeta_{2}=r_{1}-r_{2}$ :

$$
\begin{aligned}
& \int_{\mathbb{S}^{d-1}} d \omega \int d \zeta_{1} d \zeta_{2} d s_{1} d s_{2}\left(\left\langle\zeta_{1}-\zeta_{2}+2 s_{2}\right\rangle^{A}+\left\langle\zeta_{1}-\zeta_{2}-2 s_{2}\right\rangle^{A}\right) \times \\
& \times \delta\left(\tau+\frac{1}{2}\left|\frac{3 \zeta_{1}}{4}-\frac{\zeta_{2}}{4}+s_{1}+s_{2}\right|^{2}-\frac{1}{2}\left|-\frac{\zeta_{1}}{4}+\frac{3 \zeta_{2}}{4}+s_{1}-s_{2}\right|^{2}+\right. \\
& \quad+\frac{1}{2}\left|\frac{3 \zeta_{1}}{4}-\frac{\zeta_{2}}{4}-s_{1}-s_{2}\right|^{2}-\frac{1}{2}\left|-\frac{\zeta_{1}}{4}+\frac{3 \zeta_{2}}{4}-s_{1}+s_{2}\right|^{2}+ \\
& \left.\times \delta\left(-\xi_{1}+\left.\zeta_{i}\right|^{2}-\left|\xi_{i}^{\prime}\right|^{2}\right)\right) \times \\
& \times \delta\left(-\xi_{1}^{\prime}+\zeta_{2}-R_{\omega}\left(s_{2}\right)\right) \times \times \\
& \times \mid \hat{\gamma}_{0}^{(k+1)}\left(\frac{3 \zeta_{1}}{4}-\frac{\zeta_{2}}{4}+s_{1}+s_{2}, \xi_{2}, \ldots, \xi_{k}, \frac{3 \zeta_{1}}{4}-\frac{\zeta_{2}}{4}-s_{1}-s_{2},\right. \\
& \left.\frac{-\zeta_{1}}{4}+\frac{3 \zeta_{2}}{4}+s_{1}-s_{2}, \xi_{2}^{\prime}, \ldots, \xi_{k}^{\prime},-\frac{\zeta_{1}}{4}+\frac{3 \zeta_{2}}{4}-s_{1}+s_{2}\right) \mid
\end{aligned}
$$


Now we can integrate out the variables $\zeta_{1}, \zeta_{2}$ to obtain:

$$
\begin{aligned}
& \int_{\mathbb{S}^{d-1}} d \omega \int d s_{1} d s_{2}\left(\left\langle 4 s_{2}^{\|}+\xi_{1}-\xi_{1}^{\prime}\right\rangle^{A}+\left\langle-4 s_{2}^{\perp}+\xi_{1}-\xi_{1}^{\prime}\right\rangle^{A}\right) \times \\
& \times \delta\left(\tau+\frac{1}{2}\left|s_{1}+2 s_{2}^{\|}+\frac{3 \xi_{1}-\xi_{1}^{\prime}}{4}\right|^{2}-\frac{1}{2}\left|s_{1}-2 s_{2}^{\|}+\frac{3 \xi_{1}^{\prime}-\xi_{1}}{4}\right|^{2}+\right. \\
& +\frac{1}{2}\left|-s_{1}-2 s_{2}^{\perp}+\frac{3 \xi_{1}-\xi_{1}^{\prime}}{4}\right|^{2}-\frac{1}{2}\left|-s_{1}+2 s_{2}^{\perp}+\frac{3 \xi_{1}^{\prime}-\xi_{1}}{4}\right|^{2}+ \\
& \left.+\frac{1}{2} \sum_{2 \leq i \leq k}\left(\left|\xi_{i}\right|^{2}-\left|\xi_{i}^{\prime}\right|^{2}\right)\right) \times \\
& \times \mid \hat{\gamma}_{0}^{(k+1)}\left(s_{1}+2 s_{2}^{\|}+\frac{3 \xi_{1}-\xi_{1}^{\prime}}{4}, \xi_{2}, \ldots, \xi_{k},-s_{1}-2 s_{2}^{\perp}+\frac{3 \xi_{1}-\xi_{1}^{\prime}}{4},\right. \\
& \left.s_{1}-2 s_{2}^{\|}+\frac{3 \xi_{1}^{\prime}-\xi_{1}}{4}, \xi_{2}^{\prime}, \ldots, \xi_{k}^{\prime},-s_{1}+2 s_{2}^{\perp}+\frac{3 \xi_{1}^{\prime}-\xi_{1}}{4}\right) \mid
\end{aligned}
$$

where $s_{2}^{\|}=P_{\omega}\left(s_{2}\right)$ and $s_{2}^{\perp}=\left(\mathbb{I}-P_{\omega}\right)\left(s_{2}\right)$.

We want to estimate the following integral, for suitable $\alpha, \beta, \kappa, \sigma>0$ :

$$
\begin{aligned}
I^{+}(\alpha, \beta, \kappa, \sigma)=\int d \tau d \xi_{1} \ldots & d \xi_{k} d \xi_{1}^{\prime} \ldots d \xi_{k}^{\prime} \times \\
\times & \prod_{i=1}^{k}\left\{\left\langle\xi_{i}+\xi_{i}^{\prime}\right\rangle^{2 \alpha}\left\langle\xi_{i}-\xi_{i}^{\prime}\right\rangle^{2 \beta} e^{\left.2 \kappa\left\langle\xi_{i}-\xi_{i}^{\prime}\right\rangle^{\frac{1}{\sigma}}\right\} \times}\right. \\
\times & \times\left.\left(B_{1, k+1}^{+}\left[e^{\frac{1}{2} i t\left(\Delta_{X_{k+1}}-\Delta_{X_{k+1}}^{\prime}\right)} \gamma_{0}^{(k+1)}\right]\right)^{\sim}\right|^{2}
\end{aligned}
$$


Reasoning as for the loss term, if we can show that the following integral

$$
\begin{aligned}
& \int_{\mathbb{S}^{d-1}} d \omega \int d s_{1} d s_{2} \times \\
& \times \delta\left(\tau+\frac{1}{2}\left|s_{1}+2 s_{2}^{\|}+\frac{3 \xi_{1}-\xi_{1}^{\prime}}{4}\right|^{2}-\frac{1}{2}\left|s_{1}-2 s_{2}^{\|}+\frac{3 \xi_{1}^{\prime}-\xi_{1}}{4}\right|^{2}+\right. \\
& \quad+\frac{1}{2}\left|-s_{1}-2 s_{2}^{\perp}+\frac{3 \xi_{1}-\xi_{1}^{\prime}}{4}\right|^{2}-\frac{1}{2}\left|-s_{1}+2 s_{2}^{\perp}+\frac{3 \xi_{1}^{\prime}-\xi_{1}}{4}\right|^{2}+ \\
& \left.\quad+\sum_{2 \leq i \leq k}\left(\left|\xi_{i}\right|^{2}-\left|\xi_{i}^{\prime}\right|^{2}\right)\right) \times \\
& \times \frac{\left\langle\xi_{1}+\xi_{1}^{\prime}\right\rangle^{2 \alpha}\left\langle\xi_{1}-\xi_{1}^{\prime}\right\rangle^{2 \beta}\left(\left\langle 4 s_{2}^{\|}+\xi_{1}-\xi_{1}^{\prime}\right\rangle^{2 A}+\left\langle-4 s_{2}^{\perp}+\xi_{1}-\xi_{1}^{\prime}\right\rangle^{2 A}\right)}{\left\langle 2 s_{1}+\frac{\xi_{1}+\xi_{1}^{\prime}}{2}\right\rangle^{2 \alpha}\left\langle 4 s_{2}^{\|}+\xi_{1}-\xi_{1}^{\prime}\right\rangle^{2 \beta}\left\langle-2 s_{1}+\frac{\xi_{1}+\xi_{1}^{\prime}}{2}\right\rangle^{2 \alpha}\left\langle-4 s_{2}^{\perp}+\xi_{1}-\xi_{1}^{\prime}\right\rangle^{2 \beta} \times} \\
& \times e^{-2\left(\kappa_{0}-\kappa\right)\left\langle 4 s_{2}^{\|}+\xi_{1}-\xi_{1}^{\prime}\right\rangle^{\frac{1}{\sigma}}} e^{-2\left(\kappa \kappa_{0}-\kappa\right)\left\langle-4 s_{2}^{\perp}+\xi_{1}-\xi_{1}^{\prime}\right\rangle^{\frac{1}{\sigma}} \times} \times e^{2 \kappa\left(\left\langle\xi_{1}-\xi_{1}^{\prime}\right\rangle^{\frac{1}{\sigma}}-\left\langle 4 s_{2}^{\|}+\xi_{1}-\xi_{1}^{\prime}\right\rangle^{\frac{1}{\sigma}}-\left\langle-4 s_{2}^{\perp}+\xi_{1}-\xi_{1}^{\prime}\right\rangle^{\frac{1}{\sigma}}\right)} \\
& \times
\end{aligned}
$$

is bounded uniformly in $\tau, \xi_{1}, \ldots, \xi_{k}, \xi_{1}^{\prime}, \ldots, \xi_{k}^{\prime}$, then we will have the following estimate:

$$
\begin{array}{r}
\left\|B_{1, k+1}^{+}\left[e^{\frac{1}{2} i t\left(\Delta_{X_{k+1}}-\Delta_{X_{k+1}^{\prime}}\right)} \gamma_{0}^{(k+1)}\right]\right\|_{L_{t}^{2} H_{k}^{\alpha, \beta, \sigma, \kappa}} \leq \\
\leq C\left(\alpha, \beta, \sigma, \kappa, \kappa_{0}\right)\|\mathbf{b}\|_{L_{A}^{\infty}}\left\|\gamma_{0}^{(k+1)}\right\|_{H_{k+1}^{\alpha, \beta, \sigma, \kappa_{0}}}
\end{array}
$$

Before proceeding further, we must eliminate the most dangerous contribution in (87), which is the following exponential factor:

$$
e^{2 \kappa\left(\left\langle\xi_{1}-\xi_{1}^{\prime}\right\rangle^{\frac{1}{\sigma}}-\left\langle 4 s_{2}^{\|}+\xi_{1}-\xi_{1}^{\prime}\right\rangle^{\frac{1}{\sigma}}-\left\langle-4 s_{2}^{\perp}+\xi_{1}-\xi_{1}^{\prime}\right\rangle^{\frac{1}{\sigma}}\right)}
$$


We will show that this factor is in fact bounded by 1 , as long as $\sigma \geq \frac{1}{2}$. Indeed for $\sigma \geq \frac{1}{2}$ we have:

$$
\begin{aligned}
& \left\langle\xi_{1}-\xi_{1}^{\prime}\right\rangle^{\frac{1}{\sigma}}-\left\langle 4 s_{2}^{\|}+\xi_{1}-\xi_{1}^{\prime}\right\rangle^{\frac{1}{\sigma}}-\left\langle-4 s_{2}^{\perp}+\xi_{1}-\xi_{1}^{\prime}\right\rangle^{\frac{1}{\sigma}} \\
& \leq\left\langle\xi_{1}-\xi_{1}^{\prime}\right\rangle^{\frac{1}{\sigma}}-\left\langle\left(\xi_{1}-\xi_{1}^{\prime}\right)^{\perp}\right\rangle^{\frac{1}{\sigma}}-\left\langle\left(\xi_{1}-\xi_{1}^{\prime}\right)^{\|}\right\rangle^{\frac{1}{\sigma}} \\
& \leq\left(\left\langle\left(\xi_{1}-\xi_{1}^{\prime}\right)^{\|}\right\rangle^{2}+\left\langle\left(\xi_{1}-\xi_{1}^{\prime}\right)^{\perp}\right\rangle^{2}\right)^{\frac{1}{2 \sigma}}-\left\langle\left(\xi_{1}-\xi_{1}^{\prime}\right)^{\perp}\right\rangle^{\frac{1}{\sigma}}-\left\langle\left(\xi_{1}-\xi_{1}^{\prime}\right)^{\|}\right\rangle^{\frac{1}{\sigma}} \\
& \leq 0
\end{aligned}
$$

We now deal with the other exponential factors in (87), namely:

$$
e^{-2\left(\kappa_{0}-\kappa\right)\left\langle 4 s_{2}^{\|}+\xi_{1}-\xi_{1}^{\prime}\right\rangle^{\frac{1}{\sigma}}} e^{-2\left(\kappa_{0}-\kappa\right)\left\langle-4 s_{2}^{\perp}+\xi_{1}-\xi_{1}^{\prime}\right\rangle^{\frac{1}{\sigma}}}
$$

Since $\frac{1}{\sigma}>\max (0,2 A-1)$, we can always find $r \in[0,1)$ such that $\frac{r}{\sigma} \geq$ $\max (0,2 A-1+\delta)$ for a small $\delta>0$. Since $e^{u} \geq 1+u \gtrsim u^{r}$ for $u>0$, we find that if $\kappa<\kappa_{0}$ then (91) is bounded above by the following quantity:

$$
\begin{aligned}
& \left(\kappa_{0}-\kappa\right)^{-r} \times \\
& \times \min \left(\left\langle 4 s_{2}^{\|}+\xi_{1}-\xi_{1}^{\prime}\right\rangle^{-\max (0,2 A-1+\delta)},\left\langle-4 s_{2}^{\perp}+\xi_{1}-\xi_{1}^{\prime}\right\rangle^{-\max (0,2 A-1+\delta)}\right)
\end{aligned}
$$

The integral (87) is now bounded by the following integral, if $\delta>0$ is sufficiently small depending on $A, \sigma$ and $r$ : (note that this follows from the previous paragraph by considering separately $0 \leq A<\frac{1}{2}$ and $\frac{1}{2} \leq A \leq 1$ )

$$
\begin{aligned}
& \left(\kappa_{0}-\kappa\right)^{-r} \int_{\mathbb{S}^{d-1}} d \omega \int d s_{1} d s_{2} \times \\
& \times \delta\left(\tau+\frac{1}{2} \sum_{i=1}^{k}\left(\left|\xi_{i}\right|^{2}-\left|\xi_{i}^{\prime}\right|^{2}\right)+\left(4 s_{1}-R_{\omega}\left(\xi_{1}+\xi_{1}^{\prime}\right)\right) \cdot s_{2}\right) \times \\
& \times \frac{\left\langle\xi_{1}+\xi_{1}^{\prime}\right\rangle^{2 \alpha}\left\langle\xi_{1}-\xi_{1}^{\prime}\right\rangle^{2 \beta}\left(\left\langle 4 s_{2}^{\|}+\xi_{1}-\xi_{1}^{\prime}\right\rangle^{1-\delta}+\left\langle-4 s_{2}^{\perp}+\xi_{1}-\xi_{1}^{\prime}\right\rangle^{1-\delta}\right)}{\left\langle 2 s_{1}+\frac{\xi_{1}+\xi_{1}^{\prime}}{2}\right\rangle^{2 \alpha}\left\langle 4 s_{2}^{\|}+\xi_{1}-\xi_{1}^{\prime}\right\rangle^{2 \beta}\left\langle-2 s_{1}+\frac{\xi_{1}+\xi_{1}^{\prime}}{2}\right\rangle^{2 \alpha}\left\langle-4 s_{2}^{\perp}+\xi_{1}-\xi_{1}^{\prime}\right\rangle^{2 \beta}}
\end{aligned}
$$


This is in turn equivalent to the following integral:

$$
\begin{aligned}
& \left(\kappa_{0}-\kappa\right)^{-r} \times \\
& \times \int_{\mathbb{S}^{d-1}} d \omega \int d s_{2} \frac{\left\langle\xi_{1}-\xi_{1}^{\prime}\right\rangle^{2 \beta}\left(\left\langle 4 s_{2}^{\|}+\xi_{1}-\xi_{1}^{\prime}\right\rangle^{1-\delta}+\left\langle-4 s_{2}^{\perp}+\xi_{1}-\xi_{1}^{\prime}\right\rangle^{1-\delta}\right)}{\left|4 s_{2}\right|\left\langle 4 s_{2}^{\|}+\xi_{1}-\xi_{1}^{\prime}\right\rangle^{2 \beta}\left\langle-4 s_{2}^{\perp}+\xi_{1}-\xi_{1}^{\prime}\right\rangle^{2 \beta}} \times \\
& \times \int_{P} d S\left(s_{1}\right) \frac{\left\langle\xi_{1}+\xi_{1}^{\prime}\right\rangle^{2 \alpha}}{\left\langle 2 s_{1}+\frac{\xi_{1}+\xi_{1}^{\prime}}{2}\right\rangle^{2 \alpha}\left\langle-2 s_{1}+\frac{\xi_{1}+\xi_{1}^{\prime}}{2}\right\rangle^{2 \alpha}}
\end{aligned}
$$

where $P \subset \mathbb{R}^{d}$ is the following codimension one hyperplane:

$$
P=\left\{s_{1} \in \mathbb{R}^{d} \mid \tau+\frac{1}{2} \sum_{i=1}^{k}\left(\left|\xi_{i}\right|^{2}-\left|\xi_{i}^{\prime}\right|^{2}\right)+\left(4 s_{1}-R_{\omega}\left(\xi_{1}+\xi_{1}^{\prime}\right)\right) \cdot s_{2}=0\right\}
$$

Therefore we only need to show the boundedness of the following three quantities uniformly in $\xi_{1}, \xi_{1}^{\prime}, \tau$ :

$$
\begin{gathered}
I_{1}=\sup _{P \subset \mathbb{R}^{d}: \operatorname{dim} P=d-1} \int_{P} d S(s) \frac{\left\langle\xi_{1}+\xi_{1}^{\prime}\right\rangle^{2 \alpha}}{\left\langle 2 s+\frac{\xi_{1}+\xi_{1}^{\prime}}{2}\right\rangle^{2 \alpha}\left\langle-2 s+\frac{\xi_{1}+\xi_{1}^{\prime}}{2}\right\rangle^{2 \alpha}} \\
I_{2}=\int_{\mathbb{S}^{d-1}} d \omega \int_{\mathbb{R}^{d}} d s \frac{\left\langle\xi_{1}-\xi_{1}^{\prime}\right\rangle^{2 \beta}}{|4 s|\left\langle 4 s^{\|}+\xi_{1}-\xi_{1}^{\prime}\right\rangle^{2 \beta-1+\delta}\left\langle-4 s^{\perp}+\xi_{1}-\xi_{1}^{\prime}\right\rangle^{2 \beta}} \\
I_{3}=\int_{\mathbb{S}^{d-1}} d \omega \int_{\mathbb{R}^{d}} d s \frac{\left\langle\xi_{1}-\xi_{1}^{\prime}\right\rangle^{2 \beta}}{|4 s|\left\langle 4 s^{\|}+\xi_{1}-\xi_{1}^{\prime}\right\rangle^{2 \beta}\left\langle-4 s^{\perp}+\xi_{1}-\xi_{1}^{\prime}\right\rangle^{2 \beta-1+\delta}}
\end{gathered}
$$

Let us first consider the integral $I_{2}$; in what follows we will assume that $\beta>\frac{d}{2}$. Clearly, $I_{2}$ is equivalent to the following quantity:

$$
I_{2} \lesssim \int_{\mathbb{S}^{d-1}} d \omega \int_{\mathbb{R}^{d}} d s \frac{\left\langle\xi_{1}-\xi_{1}^{\prime}\right\rangle^{2 \beta}}{|s|\left\langle s^{\|}+\xi_{1}-\xi_{1}^{\prime}\right\rangle^{2 \beta-1+\delta}\left\langle s^{\perp}+\xi_{1}-\xi_{1}^{\prime}\right\rangle^{2 \beta}}
$$

Setting $W=\xi_{1}-\xi_{1}^{\prime}$, this gives:

$$
I_{2} \lesssim \int_{\mathbb{S}^{d-1}} d \omega \int_{\mathbb{R}^{d}} d s \frac{\langle W\rangle^{2 \beta}}{|s|\langle s \|+W\rangle^{2 \beta-1+\delta}\left\langle s^{\perp}+W\right\rangle^{2 \beta}}
$$

Moreover, since the integral for $|s| \leq 1$ is obviously uniformly bounded in $W$, we may instead bound the following integral:

$$
I_{2}^{\prime} \lesssim \int_{\mathbb{S}^{d-1}} d \omega \int_{\mathbb{R}^{d}} d s \frac{\langle W\rangle^{2 \beta}}{\langle s\rangle\left\langle s^{2} \|+W\right\rangle^{2 \beta-1+\delta}\left\langle s^{\perp}+W\right\rangle^{2 \beta}}
$$


Since $\left|s^{\|}\right| \leq|s|$ we have:

$$
I_{2}^{\prime} \lesssim \int_{\mathbb{S}^{d-1}} d \omega \int_{\mathbb{R}^{d}} d s \frac{\langle W\rangle^{2 \beta}}{\left\langle s^{\|}\right\rangle\left\langle s^{\|}+W\right\rangle^{2 \beta-1+\delta}\left\langle s^{\perp}+W\right\rangle^{2 \beta}}
$$

Therefore, for all large enough $|W|$,

$$
\begin{aligned}
I_{2}^{\prime} & \lesssim \int_{\mathbb{S}^{d-1}} d \omega \int_{\mathbb{R}^{d}} d s \frac{\langle W\rangle^{2 \beta}}{\left\langle s^{\|}\right\rangle\left\langle s^{\|}+W\right\rangle^{2 \beta-1+\delta}\left\langle s^{\perp}+W\right\rangle^{2 \beta}} \\
& =\int_{\mathbb{S}^{d-1}} d \omega\langle W\rangle^{2 \beta}\left(\int \frac{d s^{\|}}{\left\langle s^{\|}\right\rangle\left\langle s^{\|}+W\right\rangle^{2 \beta-1+\delta}}\right)\left(\int \frac{d s^{\perp}}{\left\langle s^{\perp}+W\right\rangle^{2 \beta}}\right) \\
& \lesssim \int_{\mathbb{S}^{d-1}} d \omega\langle W\rangle^{2 \beta}\left(\langle W\rangle^{-1}\left\langle W^{\perp}\right\rangle^{2-2 \beta-\delta} \log \langle W\rangle\right)\left(\left\langle W^{\|}\right\rangle^{d-1-2 \beta}\right)
\end{aligned}
$$

The integral over $s^{\perp}$ is estimated by a trivial computation, whereas the integral over $s^{\|}$may be estimated by considering separately the regions $\left|s^{\|}\right|<\frac{1}{2}|W|,\left|s^{\|}\right|>2|W|$, and $\frac{1}{2}|W| \leq\left|s^{\|}\right| \leq 2|W|$.

We find that $I_{2}^{\prime}$ obeys the following estimate:

$$
I_{2}^{\prime} \lesssim \int_{\mathbb{S}^{d-1}} d \omega\langle W\rangle^{2 \beta-1+\frac{1}{2} \delta}\left\langle W^{\perp}\right\rangle^{2-2 \beta-\delta}\left\langle W^{\|}\right\rangle^{d-1-2 \beta}
$$

Then we have

$$
\langle W\rangle^{2 \beta-1+\frac{1}{2} \delta} \lesssim\left\langle W^{\|}\right\rangle^{2 \beta-1+\frac{1}{2} \delta}+\left\langle W^{\perp}\right\rangle^{2 \beta-1+\frac{1}{2} \delta}
$$

Hence $I_{2}^{\prime} \lesssim I_{2}^{\prime \prime}+I_{2}^{\prime \prime \prime}$ where

$$
\begin{aligned}
& I_{2}^{\prime \prime}=\int_{\mathbb{S}^{d-1}} d \omega\left\langle W^{\perp}\right\rangle^{2-2 \beta-\delta}\left\langle W^{\|}\right\rangle^{d-2+\frac{1}{2} \delta} \\
& I_{2}^{\prime \prime \prime}=\int_{\mathbb{S}^{d-1}} d \omega\left\langle W^{\perp}\right\rangle^{1-\frac{1}{2} \delta}\left\langle W^{\|}\right\rangle^{d-1-2 \beta}
\end{aligned}
$$

Then for any $\delta$ sufficiently small and $\beta$ sufficiently large $(\beta \geq d$ is easily sufficient for small $\delta$ ), both $I_{2}^{\prime \prime}$ and $I_{2}^{\prime \prime \prime}$ may be bounded using dyadic decompositions in the angular parameter $\omega$, as follows: neglecting additive constants,

$$
\begin{aligned}
I_{2}^{\prime \prime} & \lesssim \sum_{k=1}^{\infty} \int_{\omega: 2^{-k-1}\left|W^{\|}\right| \leq\left|W^{\perp}\right|<2^{-k}|W \||} d \omega\left\langle W^{\perp}\right\rangle^{2-2 \beta-\delta}\left\langle W^{\|}\right\rangle^{d-2+\frac{1}{2} \delta} \\
& \lesssim \sum_{k=1}^{\infty} 2^{-k-1} \times\left(2^{-k}\right)^{d-2} \times\left(2^{k+1}\right)^{d-2+\frac{1}{2} \delta}<\infty
\end{aligned}
$$




$$
\begin{aligned}
I_{2}^{\prime \prime \prime} & \lesssim \sum_{k=1}^{\infty} \int_{\omega: 2^{-k-1}\left|W^{\perp}\right| \leq\left|W^{\|}\right|<2^{-k}\left|W^{\perp}\right|} d \omega\left\langle W^{\perp}\right\rangle^{1-\frac{1}{2} \delta}\left\langle W^{\|}\right\rangle^{d-1-2 \beta} \\
& \lesssim \sum_{k=1}^{\infty} 2^{-k-1} \times\left(2^{k+1}\right)^{1-\frac{1}{2} \delta}<\infty
\end{aligned}
$$

The factor of $\left(2^{-k}\right)^{d-2}$ in $I_{2}^{\prime \prime}$ comes from the Jacobian for spherical coordinates in $\mathbb{R}^{d}$.

Let us now consider the integral $I_{3}$, and assume $\beta>\frac{d}{2}$. Clearly, $I_{3}$ is equivalent to the following quantity:

$$
I_{3} \lesssim \int_{\mathbb{S}^{d-1}} d \omega \int_{\mathbb{R}^{d}} d s \frac{\left\langle\xi_{1}-\xi_{1}^{\prime}\right\rangle^{2 \beta}}{|s|\left\langle s^{\|}+\xi_{1}-\xi_{1}^{\prime}\right\rangle^{2 \beta}\left\langle s^{\perp}+\xi_{1}-\xi_{1}^{\prime}\right\rangle^{2 \beta-1+\delta}}
$$

Setting $W=\xi_{1}-\xi_{1}^{\prime}$, this gives:

$$
I_{3} \lesssim \int_{\mathbb{S}^{d-1}} d \omega \int_{\mathbb{R}^{d}} d s \frac{\langle W\rangle^{2 \beta}}{|s|\left\langle s^{\|}+W\right\rangle^{2 \beta}\left\langle s^{\perp}+W\right\rangle^{2 \beta-1+\delta}}
$$

Moreover, since the integral for $|s| \leq 1$ is obviously uniformly bounded in $W$, we may instead bound the following integral:

$$
I_{3}^{\prime} \lesssim \int_{\mathbb{S}^{d-1}} d \omega \int_{\mathbb{R}^{d}} d s \frac{\langle W\rangle^{2 \beta}}{\langle s\rangle\left\langle s^{\|}+W\right\rangle^{2 \beta}\left\langle s^{\perp}+W\right\rangle^{2 \beta-1+\delta}}
$$

Since $\left|s^{\|}\right| \leq|s|$ we have:

$$
I_{3}^{\prime} \lesssim \int_{\mathbb{S}^{d-1}} d \omega \int_{\mathbb{R}^{d}} d s \frac{\langle W\rangle^{2 \beta}}{\left\langle s^{\|}\right\rangle\left\langle s^{\|}+W\right\rangle^{2 \beta}\left\langle s^{\perp}+W\right\rangle^{2 \beta-1+\delta}}
$$

Therefore, for all large enough $|W|$,

$$
\begin{aligned}
I_{3}^{\prime} & \lesssim \int_{\mathbb{S}^{d-1}} d \omega \int_{\mathbb{R}^{d}} d s \frac{\langle W\rangle^{2 \beta}}{\left\langle s^{\|}\right\rangle\left\langle s^{\|}+W\right\rangle^{2 \beta}\left\langle s^{\perp}+W\right\rangle^{2 \beta-1+\delta}} \\
& =\int_{\mathbb{S}^{d-1}} d \omega\langle W\rangle^{2 \beta}\left(\int \frac{d s^{\|}}{\left\langle s^{\|}\right\rangle\left\langle s^{\|}+W\right\rangle^{2 \beta}}\right)\left(\int \frac{d s^{\perp}}{\left\langle s^{\perp}+W\right\rangle^{2 \beta-1+\delta}}\right) \\
& \lesssim \int_{\mathbb{S}^{d-1}} d \omega\langle W\rangle^{2 \beta}\left(\langle W\rangle^{-1}\left\langle W^{\perp}\right\rangle^{1-2 \beta} \log \langle W\rangle\right)\left(\left\langle W^{\|}\right\rangle^{d-2 \beta-\delta}\right)
\end{aligned}
$$

As before, the integral over $s^{\perp}$ is estimated by a trivial computation, whereas the integral over $s^{\|}$may be estimated by considering separately the regions $\left|s^{\|}\right|<\frac{1}{2}|W|,\left|s^{\|}\right|>2|W|$, and $\frac{1}{2}|W| \leq\left|s^{\|}\right| \leq 2|W|$.

We find that $I_{3}^{\prime}$ obeys the following estimate:

$$
I_{3}^{\prime} \lesssim \int_{\mathbb{S}^{d-1}} d \omega\langle W\rangle^{2 \beta-1+\frac{1}{2} \delta}\left\langle W^{\perp}\right\rangle^{1-2 \beta}\left\langle W^{\|}\right\rangle^{d-2 \beta-\delta}
$$


Then we have

$$
\langle W\rangle^{2 \beta-1+\frac{1}{2} \delta} \lesssim\left\langle W^{\|}\right\rangle^{2 \beta-1+\frac{1}{2} \delta}+\left\langle W^{\perp}\right\rangle^{2 \beta-1+\frac{1}{2} \delta}
$$

Hence $I_{3}^{\prime} \lesssim I_{3}^{\prime \prime}+I_{3}^{\prime \prime \prime}$ where

$$
\begin{aligned}
& I_{3}^{\prime \prime}=\int_{\mathbb{S}^{d-1}} d \omega\left\langle W^{\perp}\right\rangle^{1-2 \beta}\left\langle W^{\|}\right\rangle^{d-1-\frac{1}{2} \delta} \\
& I_{3}^{\prime \prime \prime}=\int_{\mathbb{S}^{d-1}} d \omega\left\langle W^{\perp}\right\rangle^{\frac{1}{2} \delta}\left\langle W^{\|}\right\rangle^{d-2 \beta-\delta}
\end{aligned}
$$

Then for any sufficiently small $\delta$ and $\beta>d$, both $I_{3}^{\prime \prime}$ and $I_{3}^{\prime \prime \prime}$ may be bounded using dyadic decompositions in the angular parameter $\omega$, as follows: neglecting additive constants,

$$
\begin{aligned}
I_{3}^{\prime \prime} & \lesssim \sum_{k=1}^{\infty} \int_{\omega: 2^{-k-1}\left|W^{\|}\right| \leq\left|W^{\perp}\right|<2^{-k}\left|W^{\|}\right|} d \omega\left\langle W^{\perp}\right\rangle^{1-2 \beta}\left\langle W^{\|}\right\rangle^{d-1-\frac{1}{2} \delta} \\
& \lesssim \sum_{k=1}^{\infty} 2^{-k-1} \times\left(2^{-k}\right)^{d-2} \times\left(2^{k+1}\right)^{d-1-\frac{1}{2} \delta}<\infty \\
I_{3}^{\prime \prime \prime} & \lesssim \sum_{k=1}^{\infty} \int_{\omega: 2^{-k-1}\left|W^{\perp}\right| \leq\left|W^{\|}\right|<2^{-k}\left|W^{\perp}\right|} d \omega\left\langle W^{\perp}\right\rangle^{\frac{1}{2} \delta}\left\langle W^{\|}\right\rangle^{d-2 \beta-\delta} \\
& \lesssim \sum_{k=1}^{\infty} 2^{-k-1} \times\left(2^{k+1}\right)^{\frac{1}{2} \delta}<\infty
\end{aligned}
$$

The factor of $\left(2^{-k}\right)^{d-2}$ in $I_{3}^{\prime \prime}$ comes from the Jacobian for spherical coordinates in $\mathbb{R}^{d}$.

We finally turn to $I_{1}$, which is clearly bounded by the following quantity:

$$
I_{1} \lesssim \sup _{W \in \mathbb{R}^{d}} \sup _{P \subset \mathbb{R}^{d}: \operatorname{dim} P=d-1} \int_{P} d S(s) \frac{\langle W\rangle^{2 \alpha}}{\langle s\rangle^{2 \alpha}\langle s+W\rangle^{2 \alpha}}
$$

The integrals over $P \cap\left\{|s|<\frac{1}{2}|W|\right\}, P \cap\{|s|>2|W|\}$, and $P \cap\left\{\frac{1}{2}|W| \leq|s| \leq 2|W|\right\}$ are each easily bounded uniformly in $W$ as long as $\alpha>\frac{d-1}{2}$.

To summarize, as long as $\alpha>\frac{d-1}{2}, \beta>d$, and $\max (0,2 A-1)<\frac{1}{\sigma} \leq 2$, then for $r \in[0,1)$ such that $\frac{r}{\sigma} \geq \max (0,2 A-1+\delta)$ for a small $\delta>0$ we have for any $\kappa_{0}>\kappa>0$ the following estimate:

$$
\begin{array}{r}
\left\|B_{i, k+1}^{+}\left[e^{\frac{1}{2} i t\left(\Delta_{X_{k+1}}-\Delta_{X_{k+1}^{\prime}}\right)} \gamma_{0}^{(k+1)}\right]\right\|_{L_{t}^{2} H_{k}^{\alpha, \beta, \sigma, \kappa}} \leq \\
\leq C(\alpha, \beta, \sigma, r)\|\mathbf{b}\|_{L_{A}^{\infty}}\left(\kappa_{0}-\kappa\right)^{-\frac{1}{2} r}\left\|\gamma_{0}^{(k+1)}\right\|_{H_{k+1}^{\alpha, \beta, \sigma, \kappa}}
\end{array}
$$




\section{Proof of Theorem 2.2}

Formally speaking, solutions of Boltzmann's equation are factorized solutions of the Boltzmann hierarchy, i.e. $\gamma^{(k)}=\gamma^{\otimes k}$. We use the notation

$$
\begin{gathered}
\Delta_{ \pm}^{(k)}=\Delta_{X_{k}}-\Delta_{X_{k}^{\prime}} \\
\Delta_{ \pm}=\Delta_{ \pm}^{(1)}
\end{gathered}
$$

Then if $B_{k+1}=\sum_{i=1}^{k}\left(B_{i, k+1}^{+}-B_{i, k+1}^{-}\right)$, the Boltzmann hierarchy in integral form reads as follows:

$$
\gamma^{(k)}(t)=e^{\frac{1}{2} i t \Delta_{ \pm}^{(k)}} \gamma^{(k)}(0)-i \int_{0}^{t} e^{\frac{1}{2} i\left(t-t_{1}\right) \Delta_{ \pm}^{(k)}} B_{k+1} \gamma^{(k+1)}\left(t_{1}\right) d t_{1}
$$

Let us assume $\gamma^{(k)}=\gamma^{\otimes k}$ for all $k \in \mathbb{N}$ and consider the Boltzmann hierarchy for $k=1,2$ :

$$
\begin{gathered}
\gamma(t)=e^{\frac{1}{2} i t \Delta_{ \pm}} \gamma(0)-i \int_{0}^{t} e^{\frac{1}{2} i\left(t-t_{1}\right) \Delta_{ \pm}} B_{2}\left(\gamma^{\otimes 2}\right)\left(t_{1}\right) d t_{1} \\
(\gamma \otimes \gamma)(t)=e^{\frac{1}{2} i t \Delta_{ \pm}^{(2)}}(\gamma \otimes \gamma)(0)-i \int_{0}^{t} e^{\frac{1}{2} i\left(t-t_{1}\right) \Delta_{ \pm}^{(2)}} B_{3}\left(\gamma^{\otimes 3}\right)\left(t_{1}\right) d t_{1}
\end{gathered}
$$

Now we apply the operator $B_{2}$ to the second equation, thereby obtaining the following system:

$$
\begin{gathered}
\gamma(t)=e^{\frac{1}{2} i t \Delta_{ \pm}} \gamma(0)-i \int_{0}^{t} e^{\frac{1}{2} i\left(t-t_{1}\right) \Delta_{ \pm}} B_{2}\left(\gamma^{\otimes 2}\right)\left(t_{1}\right) d t_{1} \\
B_{2}\left(\gamma^{\otimes 2}\right)(t)= \\
=B_{2}\left(e^{\frac{1}{2} i t \Delta_{ \pm}^{(2)}}\left(\gamma^{\otimes 2}\right)(0)\right)-i \int_{0}^{t} B_{2}\left[e^{\frac{1}{2} i\left(t-t_{1}\right) \Delta_{ \pm}^{(2)}} B_{3}\left(\gamma^{\otimes 3}\right)\left(t_{1}\right)\right] d t_{1}
\end{gathered}
$$

Let us observe that $B\left(\gamma_{1}, \gamma_{2}\right)=B_{2}\left(\gamma_{1} \otimes \gamma_{2}\right)$. Therefore if we define $\zeta(t)=$ $B(\gamma(t), \gamma(t))$ then we obtain the following system of equations for the pair $(\gamma, \zeta)$ :

$$
\begin{gathered}
\gamma(t)=e^{\frac{1}{2} i t \Delta_{ \pm}} \gamma(0)-i \int_{0}^{t} e^{\frac{1}{2} i\left(t-t_{1}\right) \Delta_{ \pm}} \zeta\left(t_{1}\right) d t_{1} \\
\zeta(t)=B\left(e^{\frac{1}{2} i t \Delta_{ \pm}} \gamma(0), e^{\frac{1}{2} i t \Delta_{ \pm}} \gamma(0)\right)+ \\
+(-i) \int_{0}^{t} B\left(e^{\frac{1}{2} i\left(t-t_{1}\right) \Delta_{ \pm}} \gamma\left(t_{1}\right), e^{\frac{1}{2} i\left(t-t_{1}\right) \Delta_{ \pm}} \zeta\left(t_{1}\right)\right) d t_{1}+ \\
+(-i) \int_{0}^{t} B\left(e^{\frac{1}{2} i\left(t-t_{1}\right) \Delta_{ \pm}} \zeta\left(t_{1}\right), e^{\frac{1}{2} i\left(t-t_{1}\right) \Delta_{ \pm}} \gamma\left(t_{1}\right)\right) d t_{1}
\end{gathered}
$$

We will solve this simultaneous system of equations for $(\gamma(t), \zeta(t))$ on a small time interval $[0, T]$ by Picard iteration, using the following norm:

$$
\|(\gamma, \zeta)\|=T^{\frac{1}{2}(1-r)}\|\| \gamma(t)\left\|_{H^{\alpha, \beta, \sigma, \kappa-\lambda t}}\right\|_{L_{T}^{\infty}}+\|\| \zeta(t)\left\|_{H^{\alpha, \beta, \sigma, \kappa-\lambda t}}\right\|_{L_{T}^{1}}
$$


Here we have fixed some $r \in[0,1)$ as in the statement of Proposition 3.1] The key result we will use is that Proposition 3.1 implies the following bilinear estimates:

$$
\begin{aligned}
\| B\left(e^{\frac{1}{2} i t \Delta_{ \pm}} \gamma_{0,1}, e^{\frac{1}{2} i t \Delta_{ \pm}} \gamma_{0,2}\right) & \|_{L_{t}^{2} H^{\alpha, \beta, \sigma, \kappa_{1}}} \leq \\
\leq & C\left(1+\left(\kappa_{0}-\kappa_{1}\right)^{-\frac{1}{2} r}\right)\left\|\gamma_{0,1}\right\|_{H^{\alpha, \beta, \sigma, \kappa_{0}}}\left\|\gamma_{0,2}\right\|_{H^{\alpha, \beta, \sigma, \kappa_{0}}}
\end{aligned}
$$

To set up the fixed point iteration, we fix the initial data $\gamma_{0} \in H^{\alpha, \beta, \sigma, \kappa}$ and define the map $\Phi=\left(\Phi_{1}, \Phi_{2}\right)(\gamma, \zeta)$ as follows:

$$
\begin{gathered}
{\left[\Phi_{1}(\gamma, \zeta)\right](t)=e^{\frac{1}{2} i t \Delta_{ \pm}} \gamma_{0}-i \int_{0}^{t} e^{\frac{1}{2} i\left(t-t_{1}\right) \Delta_{ \pm}} \zeta\left(t_{1}\right) d t_{1}} \\
{\left[\Phi_{2}(\gamma, \zeta)\right](t)=B\left(e^{\frac{1}{2} i t \Delta_{ \pm}} \gamma_{0}, e^{\frac{1}{2} i t \Delta_{ \pm}} \gamma_{0}\right)+} \\
+(-i) \int_{0}^{t} B\left(e^{\frac{1}{2} i\left(t-t_{1}\right) \Delta_{ \pm}} \gamma\left(t_{1}\right), e^{\frac{1}{2} i\left(t-t_{1}\right) \Delta_{ \pm}} \zeta\left(t_{1}\right)\right) d t_{1}+ \\
+(-i) \int_{0}^{t} B\left(e^{\frac{1}{2} i\left(t-t_{1}\right) \Delta_{ \pm}} \zeta\left(t_{1}\right), e^{\frac{1}{2} i\left(t-t_{1}\right) \Delta_{ \pm}} \gamma\left(t_{1}\right)\right) d t_{1}
\end{gathered}
$$

We wish to solve the equation $(\gamma, \zeta)=\Phi(\gamma, \zeta)$.

First, using (134) and the fact that the propagator $e^{\frac{1}{2} i t \Delta_{ \pm}}$preserves the space $H^{\alpha, \beta, \sigma, \kappa}$, along with the embedding $H^{\alpha, \beta, \sigma, \kappa_{0}} \subset H^{\alpha, \beta, \sigma, \kappa_{1}}$ for $\kappa_{0}>$ $\kappa_{1}>0$, we easily obtain:

$$
\|\|\left[\Phi_{1}(\gamma, \zeta)\right](t)\left\|_{H^{\alpha, \beta, \sigma, \kappa-\lambda t}}\right\|_{L_{T}^{\infty}} \leq\left\|\gamma_{0}\right\|_{H^{\alpha, \beta, \sigma, \kappa}}+\|\| \zeta(t)\left\|_{H^{\alpha, \beta, \sigma, \kappa-\lambda t}}\right\|_{L_{T}^{1}}
$$

We now turn to $\Phi_{2}$. We begin by estimating the first term on the right hand side of (135). We will use a dyadic decomposition in time:

$$
\begin{aligned}
& \|\| B\left(e^{\frac{1}{2} i t \Delta_{ \pm}} \gamma_{0}, e^{\frac{1}{2} i t \Delta_{ \pm}} \gamma_{0}\right)\left\|_{H^{\alpha, \beta, \sigma, \kappa-\lambda t}}\right\|_{L_{T}^{1}}= \\
& =\sum_{m=0}^{\infty} \int_{2^{-m-1} T<t \leq 2^{-m} T}\left\|B\left(e^{\frac{1}{2} i t \Delta_{ \pm}} \gamma_{0}, e^{\frac{1}{2} i t \Delta_{ \pm}} \gamma_{0}\right)\right\|_{H^{\alpha, \beta, \sigma, \kappa-\lambda t}} d t \\
& \leq \sum_{m=0}^{\infty} \int_{2^{-m-1} T<t \leq 2^{-m} T}\left\|B\left(e^{\frac{1}{2} i t \Delta_{ \pm}} \gamma_{0}, e^{\frac{1}{2} i t \Delta_{ \pm}} \gamma_{0}\right)\right\|_{H^{\alpha, \beta, \sigma, \kappa-\lambda 2^{-m-1} T}} d t
\end{aligned}
$$

Now apply the Cauchy-Schwarz inequality, followed by (133). We implicitly assume $\lambda T<1$, which is acceptable because we only want to address small 
times $T$ in any case.

$$
\begin{aligned}
& \|\| B\left(e^{\frac{1}{2} i t \Delta_{ \pm}} \gamma_{0}, e^{\frac{1}{2} i t \Delta_{ \pm}} \gamma_{0}\right)\left\|_{H^{\alpha, \beta, \sigma, \kappa-\lambda t}}\right\|_{L_{T}^{1}} \leq \\
& \leq \sum_{m=0}^{\infty}\left(2^{-m-1} T\right)^{\frac{1}{2}}\left\|B\left(e^{\frac{1}{2} i t \Delta_{ \pm}} \gamma_{0}, e^{\frac{1}{2} i t \Delta_{ \pm}} \gamma_{0}\right)\right\|_{L_{t}^{2} H^{\alpha, \beta, \sigma, \kappa-\lambda 2^{-m-1} T}} \\
& \leq \sum_{m=0}^{\infty}\left(2^{-m-1} T\right)^{\frac{1}{2}} \frac{C}{\left(\lambda 2^{-m-1} T\right)^{\frac{1}{2} r}}\left\|\gamma_{0}\right\|_{H^{\alpha, \beta, \sigma, \kappa}}^{2} \\
& \leq C \lambda^{-\frac{1}{2} r} T^{\frac{1}{2}(1-r)}\left(\sum_{m=0}^{\infty} 2^{-\frac{1}{2} m(1-r)}\right)\left\|\gamma_{0}\right\|_{H^{\alpha, \beta, \sigma, \kappa}}^{2}
\end{aligned}
$$

We now estimate the second term on the right hand side of (135); the third term is handled similarly. We will employ a dyadic decomposition in $t-t_{1}$ and apply Cauchy-Schwarz and (133) as before.

$$
\begin{aligned}
& \|\| \int_{0}^{t} B\left(e^{\frac{1}{2} i\left(t-t_{1}\right) \Delta_{ \pm}} \gamma\left(t_{1}\right), e^{\frac{1}{2} i\left(t-t_{1}\right) \Delta_{ \pm}} \zeta\left(t_{1}\right)\right) d t_{1}\left\|_{H^{\alpha, \beta, \sigma, \kappa-\lambda t}}\right\|_{L_{T}^{1}} \leq \\
& \leq \sum_{m=0}^{\infty} \int_{0}^{T} d t_{1} \int_{t_{1}+2^{-m-1} T<t \leq t_{1}+2^{-m} T} d t \times \\
& \times\left\|B\left(e^{\frac{1}{2} i\left(t-t_{1}\right) \Delta_{ \pm}} \gamma\left(t_{1}\right), e^{\frac{1}{2} i\left(t-t_{1}\right) \Delta_{ \pm}} \zeta\left(t_{1}\right)\right)\right\|_{H^{\alpha, \beta, \sigma, \kappa-\lambda t}} \\
& \leq \sum_{m=0}^{\infty} \int_{0}^{T} d t_{1} \int_{t_{1}+2^{-m-1} T<t \leq t_{1}+2^{-m} T} d t \times \\
& \times\left\|B\left(e^{\frac{1}{2} i\left(t-t_{1}\right) \Delta_{ \pm}} \gamma\left(t_{1}\right), e^{\frac{1}{2} i\left(t-t_{1}\right) \Delta_{ \pm}} \zeta\left(t_{1}\right)\right)\right\|_{\left.H^{\alpha, \beta, \sigma, \kappa-\lambda\left(t_{1}+2-m-1\right.} T\right)} \\
& \leq \sum_{m=0}^{\infty}\left(2^{-m-1} T\right)^{\frac{1}{2}} \int_{0}^{T} d t_{1} \times \\
& \times\left\|B\left(e^{\frac{1}{2} i\left(t-t_{1}\right) \Delta_{ \pm}} \gamma\left(t_{1}\right), e^{\frac{1}{2} i\left(t-t_{1}\right) \Delta_{ \pm}} \zeta\left(t_{1}\right)\right)\right\|_{L_{t}^{2} H^{\alpha, \beta, \sigma, \kappa-\lambda\left(t_{1}+2^{-m-1} T\right)}} \\
& \leq \sum_{m=0}^{\infty}\left(2^{-m-1} T\right)^{\frac{1}{2}} \times C\left(\lambda 2^{-m-1} T\right)^{-\frac{1}{2} r} \times \\
& \times \int_{0}^{T} d t_{1}\left\|\gamma\left(t_{1}\right)\right\|_{H^{\alpha, \beta, \sigma, \kappa-\lambda t_{1}}}\left\|\zeta\left(t_{1}\right)\right\|_{H^{\alpha, \beta, \sigma, \kappa-\lambda t_{1}}} \\
& \leq C \lambda^{-\frac{1}{2} r} T^{\frac{1}{2}(1-r)}\left(\sum_{m=0}^{\infty} 2^{-\frac{1}{2} m(1-r)}\right) \times \\
& \times\|\| \gamma(t)\left\|_{H^{\alpha, \beta, \sigma, \kappa-\lambda t}}\right\|_{L_{T}^{\infty}}\|\| \zeta(t)\left\|_{H^{\alpha, \beta, \sigma, \kappa-\lambda t}}\right\|_{L_{T}^{1}}
\end{aligned}
$$


We can finally conclude the following estimate for $\Phi_{2}$ :

$$
\begin{aligned}
& \|\|\left[\Phi_{2}(\gamma, \zeta)\right](t)\left\|_{H^{\alpha, \beta, \sigma, \kappa-\lambda t}}\right\|_{L_{T}^{1}} \leq C \lambda^{-\frac{1}{2} r} T^{\frac{1}{2}(1-r)}\left(\sum_{m=0}^{\infty} 2^{-\frac{1}{2} m(1-r)}\right) \times \\
& \times\left(\left\|\gamma_{0}\right\|_{H^{\alpha, \beta, \sigma, \kappa}}^{2}+\|\| \gamma(t)\left\|_{H^{\alpha, \beta, \sigma, \kappa-\lambda t}}\right\|_{L_{T}^{\infty}}\|\| \zeta(t) \|_{H^{\alpha, \beta, \sigma, \kappa-\lambda t} \|_{L_{T}^{1}}}\right)
\end{aligned}
$$

Combining (136) and (137), and defining $C_{\lambda, r}=C \lambda^{-\frac{1}{2} r} \sum_{m=0}^{\infty} 2^{-\frac{1}{2} m(1-r)}$, we obtain:

$$
\begin{array}{r}
\|\Phi(\gamma, \zeta)\| \leq T^{\frac{1}{2}(1-r)}\left\|\gamma_{0}\right\|_{H^{\alpha, \beta, \sigma, \kappa}}+C_{\lambda, r} T^{\frac{1}{2}(1-r)}\left\|\gamma_{0}\right\|_{H^{\alpha, \beta, \sigma, \kappa}}^{2}+ \\
+T^{\frac{1}{2}(1-r)}\|(\gamma, \zeta)\|+C_{\lambda, r}\|(\gamma, \zeta)\|^{2}
\end{array}
$$

By a completely analogous argument, we obtain the following continuity bound:

$$
\begin{aligned}
& \left\|\Phi\left(\gamma_{1}, \zeta_{1}\right)-\Phi\left(\gamma_{2}, \zeta_{2}\right)\right\| \leq \\
& \quad \leq\left(T^{\frac{1}{2}(1-r)}+4 C_{\lambda, r}\left(\left\|\left(\gamma_{1}, \zeta_{1}\right)\right\|+\left\|\left(\gamma_{2}, \zeta_{2}\right)\right\|\right)\right)\left\|\left(\gamma_{1}, \zeta_{1}\right)-\left(\gamma_{2}, \zeta_{2}\right)\right\|
\end{aligned}
$$

Combining (138) and (139), and applying the Banach fixed point theorem, we conclude the existence and uniqueness of a solution to the fixed point equation $(\gamma, \zeta)=\Phi(\gamma, \zeta)$ once $T$ is chosen sufficiently small depending only on $\left\|\gamma_{0}\right\|_{H^{\alpha, \beta, \sigma, \kappa}}$. This gives us uniqueness under the assumption that $\|(\gamma, \zeta)\|$ is small, but in fact for any solution we can apply (138) and a standard continuity argument to conclude that $\|(\gamma, \zeta)\|$ is necessarily small if $T$ is small, as long as it is finite for some positive $T$. The estimate (33) follows directly from (138).

Finally we remark that if $A \in\left[0, \frac{1}{2}\right)$ then we may take $r=0$, so that $C_{\lambda, r}$ loses its dependence on $\lambda$; hence, we are allowed to take $\lambda=0$ and we can permit any $\kappa \in[0, \infty)$. The rest of the proof proceeds in exactly the same manner.

\section{Proof of Theorem 2.3}

Theorem 2.3 follows from Proposition 3.1 combined with the arguments of Chen and Pavlović, [5], which in turn rely on the combinatorial arguments of Erdös-Schlein-Yau, [8-10], in the boardgame representation given by Klainerman and Machedon in [17]. We outline the proof here for the reader's convenience.

To begin, we point out that the Boltzmann hierarchy may be written in vector integral form as follows:

$$
\Gamma(t)=e^{\frac{1}{2} i t \hat{\Delta}_{ \pm}} \Gamma(0)-i \int_{0}^{t} e^{\frac{1}{2} i\left(t-t_{1}\right) \hat{\Delta}_{ \pm}} B \Gamma\left(t_{1}\right) d t_{1}
$$


where $\hat{\Delta}_{ \pm} \Gamma=\left\{\left(\Delta_{X_{k}}-\Delta_{X_{k}^{\prime}}\right) \gamma^{(k)}\right\}_{k \in \mathbb{N}}$ and $B \Gamma=\left\{B_{k+1} \gamma^{(k+1)}\right\}_{k \in \mathbb{N}^{*}}$. We can apply $B$ to both sides to yield a closed equation for $B \Gamma$ :

$$
B \Gamma(t)=B\left[e^{\frac{1}{2} i t \hat{\Delta}_{ \pm}} \Gamma(0)\right]-i \int_{0}^{t} B\left[e^{\frac{1}{2} i\left(t-t_{1}\right) \hat{\Delta}_{ \pm}} B \Gamma\left(t_{1}\right)\right] d t_{1}
$$

Letting $\Xi=B \Gamma$, we conclude that the pair $(\Gamma, \Xi)$ satisfies the following system of equations:

$$
\begin{gathered}
\Gamma(t)=e^{\frac{1}{2} i t \hat{\Delta}_{ \pm}} \Gamma(0)-i \int_{0}^{t} e^{\frac{1}{2} i\left(t-t_{1}\right) \hat{\Delta}_{ \pm}} \Xi\left(t_{1}\right) d t_{1} \\
\Xi(t)=B\left[e^{\frac{1}{2} i t \hat{\Delta}_{ \pm}} \Gamma(0)\right]-i \int_{0}^{t} B\left[e^{\frac{1}{2} i\left(t-t_{1}\right) \hat{\Delta}_{ \pm}} \Xi\left(t_{1}\right)\right] d t_{1}
\end{gathered}
$$

and this system is equivalent to the original Boltzmann hierarchy.

Since (143) is a closed equation for $\Xi$, we proceed in two steps. First we solve (143) on a small time interval $[0, T]$ by Picard iteration; then, we establish that the right-hand side of (142) is indeed well-defined in the correct functional space. The proof proceeds by iterating the Duhamel formula (143),$k$ times for the $k$ th component, and applying the combinatorial methods of Erdös, Schlein and Yau, 8 10], expressed in boardgame form by Klainerman and Machedon [17. Then we conclude by applying Proposition 3.1 inductively to bound all the terms (which are now $\mathcal{O}\left(C^{k}\right)$ in number instead of (even more than) $\mathcal{O}(k !)$ due to the combinatorial methods of [8-10]). The precise details are written out in [5] for the interested reader.

Remark. Note that if $A \geq \frac{1}{2}$, then the combinatorial methods of [8 10], and the reformulation in terms of a boardgame argument [17, all still apply at the formal level. However, the termwise estimates of [5] no longer apply uniformly across general re-ordering of collision times. This is simply not an issue when $A<\frac{1}{2}$ since no time-dependent loss of weight is required in that case. Most likely, if LWP holds at all for the hard sphere Boltzmann hierarchy for the spaces we consider, then completely new estimates (different from Proposition 3.1) will be required.

\section{Appendix A. Inverse Wigner Transform of the Boltzmann EQUATION AND BolTZMANN HiERARCHY}

We begin with the Boltzmann hierarchy.

Proposition A.1. Let $f^{(k)} \in L^{1}\left([0, T], L^{2}\left(\mathbb{R}^{d k} \times \mathbb{R}^{d k}\right)\right)$ and let $\gamma^{(k)}$ denote the inverse Wigner transform of $f^{(k)}$. Then if

$$
\left(\partial_{t}+V_{k} \cdot \nabla_{X_{k}}\right) f^{(k)}=g^{(k)}
$$


holds in the sense of distributions, then we have

$$
\begin{aligned}
\left(i \partial_{t}+\frac{1}{2}\left(\Delta_{X_{k}}-\Delta_{X_{k}^{\prime}}\right)\right) & \gamma^{(k)}\left(t, X_{k}, X_{k}^{\prime}\right)= \\
= & i \int_{\mathbb{R}^{d k}} g^{(k)}\left(t, \frac{X_{k}+X_{k}^{\prime}}{2}, V_{k}\right) e^{i V_{k} \cdot\left(X_{k}-X_{k}^{\prime}\right)} d V_{k}
\end{aligned}
$$

in the sense of distributions.

Proof. We have

$$
\begin{aligned}
& i \partial_{t} \gamma^{(k)}\left(t, X_{k}, X_{k}^{\prime}\right)=i \int_{\mathbb{R}^{d k}}\left(\partial_{t} f^{(k)}\right)\left(t, \frac{X_{k}+X_{k}^{\prime}}{2}, V_{k}\right) e^{i V_{k} \cdot\left(X_{k}-X_{k}^{\prime}\right)} d V_{k} \\
& =i \int_{\mathbb{R}^{d k}}\left(-V_{k} \cdot \nabla_{X_{k}} f^{(k)}+g^{(k)}\right)\left(t, \frac{X_{k}+X_{k}^{\prime}}{2}, V_{k}\right) e^{i V_{k} \cdot\left(X_{k}-X_{k}^{\prime}\right)} d V_{k}
\end{aligned}
$$

Consider the transport term alone.

$$
\begin{aligned}
& i \int\left(-V_{k} \cdot \nabla_{X_{k}} f^{(k)}\right)\left(t, \frac{X_{k}+X_{k}^{\prime}}{2}, V_{k}\right) e^{i V_{k} \cdot\left(X_{k}-X_{k}^{\prime}\right)} d V_{k} \\
& =-i \int V_{k} \cdot\left(\nabla_{X_{k}}+\nabla_{X_{k}^{\prime}}\right)\left[f(t)\left(t, \frac{X_{k}+X_{k}^{\prime}}{2}, V_{k}\right)\right] e^{i V_{k} \cdot\left(X_{k}-X_{k}^{\prime}\right)} d V_{k} \\
& =-\int\left(\nabla_{X_{k}}+\nabla_{X_{k}^{\prime}}\right)\left[f^{(k)}\left(t, \frac{X_{k}+X_{k}^{\prime}}{2}, v\right)\right] \cdot i V_{k} e^{i V_{k} \cdot\left(X_{k}-X_{k}^{\prime}\right)} d V_{k} \\
& =-\left(\nabla_{X_{k}}+\nabla_{X_{k}^{\prime}}\right) \cdot \int f^{(k)}\left(t, \frac{X_{k}+X_{k}^{\prime}}{2}, V_{k}\right) i V_{k} e^{i V_{k} \cdot\left(X_{k}-X_{k}^{\prime}\right)} d V_{k} \\
& =-\left(\nabla_{X_{k}}+\nabla_{X_{k}^{\prime}}\right) \cdot \int f^{(k)}\left(t, \frac{X_{k}+X_{k}^{\prime}}{2}, V_{k}\right) \frac{\nabla_{X_{k}}-\nabla_{X_{k}^{\prime}}}{2} e^{i V_{k} \cdot\left(X_{k}-X_{k}^{\prime}\right)} d V_{k} \\
& =-\left(\nabla_{X_{k}}+\nabla_{X_{k}^{\prime}}\right) \cdot \frac{\nabla_{X_{k}}-\nabla_{X_{k}^{\prime}}}{2} f^{(k)}\left(t, \frac{X_{k}+X_{k}^{\prime}}{2}, V_{k}\right) e^{i V_{k} \cdot\left(X_{k}-X_{k}^{\prime}\right)} d V_{k} \\
& =-\frac{1}{2}\left(\Delta_{X_{k}}-\Delta_{X_{k}^{\prime}}\right) \gamma^{(k)}\left(t, X_{k}, X_{k}^{\prime}\right)
\end{aligned}
$$

Therefore,

$$
\begin{aligned}
& \left(i \partial_{t}+\frac{1}{2}\left(\Delta_{X_{k}}-\Delta_{X_{k}^{\prime}}\right)\right) \gamma^{(k)}\left(t, X_{k}, X_{k}^{\prime}\right)= \\
& \quad=i \int_{\mathbb{R}^{d k}} g^{(k)}\left(t, \frac{X_{k}+X_{k}^{\prime}}{2}, V_{k}\right) e^{i V_{k} \cdot\left(X_{k}-X_{k}^{\prime}\right)} d V_{k}
\end{aligned}
$$


Proposition A.2. Let $f^{(k+1)}\left(X_{k+1}, V_{k+1}\right)$ be a Schwartz function, and let $\gamma^{(k+1)}$ denote its inverse Wigner transform. Then

$$
\begin{aligned}
& i \int C_{1, k+1}^{-} f^{(k+1)}\left(\frac{X_{k}+X_{k}^{\prime}}{2}, V_{k}\right) e^{i V_{k} \cdot\left(X_{k}-X_{k}^{\prime}\right)} d V_{k}= \\
& =\frac{i}{2^{2 d} \pi^{d}} \int_{\mathbb{S}^{d-1}} d \omega \int_{\mathbb{R}^{d}} d z \hat{\mathbf{b}}^{\omega}\left(\frac{z}{2}\right) \times \\
& \quad \times \gamma^{(k+1)}\left(x_{1}-\frac{z}{4}, X_{2: k}, \frac{x_{1}+x_{1}^{\prime}}{2}+\frac{z}{4}, x_{1}^{\prime}+\frac{z}{4}, X_{2: k}^{\prime}, \frac{x_{1}+x_{1}^{\prime}}{2}-\frac{z}{4}\right)
\end{aligned}
$$

and

$$
\begin{aligned}
& i \int C_{1, k+1}^{+} f^{(k+1)}\left(\frac{X_{k}+X_{k}^{\prime}}{2}, V_{k}\right) e^{i V_{k} \cdot\left(X_{k}-X_{k}^{\prime}\right)} d V_{k}= \\
& =\frac{i}{2^{2 d} \pi^{d}} \int_{\mathbb{S}^{d-1}} d \omega \int_{\mathbb{R}^{d}} d z \hat{\mathbf{b}}^{\omega}\left(\frac{z}{2}\right) \times \\
& \times \gamma^{(k+1)}\left(x_{1}-\frac{1}{2} P_{\omega}\left(x_{1}-x_{1}^{\prime}\right)-\frac{R_{\omega}(z)}{4}, X_{2: k},\right. \\
& \frac{x_{1}+x_{1}^{\prime}}{2}+\frac{1}{2} P_{\omega}\left(x_{1}-x_{1}^{\prime}\right)+\frac{R_{\omega}(z)}{4}, \\
& x_{1}^{\prime}+\frac{1}{2} P_{\omega}\left(x_{1}-x_{1}^{\prime}\right)+\frac{R_{\omega}(z)}{4}, X_{2: k}^{\prime}, \\
& \left.\frac{x_{1}+x_{1}^{\prime}}{2}-\frac{1}{2} P_{\omega}\left(x_{1}-x_{1}^{\prime}\right)-\frac{R_{\omega}(z)}{4}\right)
\end{aligned}
$$


Proof. Consider the loss term.

$$
\begin{aligned}
& i \int C_{1, k+1}^{-} f^{(k+1)}\left(\frac{X_{k}+X_{k}^{\prime}}{2}, V_{k}\right) e^{i V_{k} \cdot\left(X_{k}-X_{k}^{\prime}\right)} d V_{k}= \\
& =i \int d V_{k+1} d \omega \mathbf{b}\left(\left|v_{k+1}-v_{1}\right|, \omega \cdot \frac{v_{k+1}-v_{1}}{\left|v_{k+1}-v_{1}\right|}\right) \times \\
& \quad \times e^{i V_{k} \cdot\left(X_{k}-X_{k}^{\prime}\right)} f^{(k+1)}\left(\frac{X_{k}+X_{k}^{\prime}}{2}, \frac{x_{1}+x_{1}^{\prime}}{2}, V_{k}, v_{k+1}\right) \\
& =i \frac{1}{(2 \pi)^{d(k+1)}} \int d V_{k+1} d Y_{k+1} d \omega \mathbf{b}\left(\left|v_{k+1}-v_{1}\right|, \omega \cdot \frac{v_{k+1}-v_{1}}{\left|v_{k+1}-v_{1}\right|}\right) \times \\
& \left.\times e^{i V_{k} \cdot\left(X_{k}-X_{k}^{\prime}\right)} e^{-i V_{k+1} \cdot Y_{k+1} \times}, \frac{X_{k}+X_{k}^{\prime}-Y_{k}}{2}, \frac{x_{1}+x_{1}^{\prime}-y_{k+1}}{2}\right) \\
& \times \gamma^{(k+1)}\left(\frac{X_{k}+X_{k}^{\prime}+Y_{k}}{2}, \frac{x_{1}+x_{1}^{\prime}+y_{k+1}}{2}, \frac{X_{k+1}-v_{1}}{\left|v_{k+1}-v_{1}\right|}\right) \times \\
& =i \frac{1}{(2 \pi)^{2 d}} \int d v_{1} d v_{k+1} d y_{1} d y_{k+1} d \omega \mathbf{b}\left(\left|v_{k+1}-v_{1}\right|, \omega \cdot x^{\prime} e^{-i v_{k+1} \cdot y_{k+1}} \times\right. \\
& \times e^{i v_{1} \cdot\left(x_{1}-x_{1}^{\prime}-y_{1}\right)} \\
& \times \gamma^{(k+1)}\left(\frac{x_{1}+x_{1}^{\prime}+y_{1}}{2}, X_{2: k}, \frac{x_{1}+x_{1}^{\prime}+y_{k+1}}{2},\right. \\
& 2
\end{aligned}
$$

Use the change of variables $u_{1}=\frac{1}{2}\left(v_{k+1}+v_{1}\right), u_{2}=\frac{1}{2}\left(v_{k+1}-v_{1}\right)$.

$$
\begin{aligned}
& i \int C_{1, k+1}^{-} f^{(k+1)}\left(\frac{X_{k}+X_{k}^{\prime}}{2}, V_{k}\right) e^{i V_{k} \cdot\left(X_{k}-X_{k}^{\prime}\right)} d V_{k}= \\
& =i \frac{1}{2^{d} \pi^{2 d}} \int d u_{1} d u_{2} d y_{1} d y_{k+1} d \omega \mathbf{b}\left(2\left|u_{2}\right|, \omega \cdot \frac{u_{2}}{\left|u_{2}\right|}\right) \times \\
& \quad \times e^{i u_{1} \cdot\left(x_{1}-x_{1}^{\prime}-y_{1}-y_{k+1}\right)} e^{-i u_{2} \cdot\left(x_{1}-x_{1}^{\prime}-y_{1}+y_{k+1}\right)} \times \\
& \quad \times \gamma^{(k+1)}\left(\frac{x_{1}+x_{1}^{\prime}+y_{1}}{2}, X_{2: k}, \frac{x_{1}+x_{1}^{\prime}+y_{k+1}}{2},\right. \\
& \left.\frac{x_{1}+x_{1}^{\prime}-y_{1}}{2}, X_{2: k}^{\prime}, \frac{x_{1}+x_{1}^{\prime}-y_{k+1}}{2}\right)
\end{aligned}
$$


Now let $w=x_{1}-x_{1}^{\prime}-y_{1}-y_{k+1}, z=x_{1}-x_{1}^{\prime}-y_{1}+y_{k+1}$.

$$
\begin{aligned}
& i \int C_{1, k+1}^{-} f^{(k+1)}\left(\frac{X_{k}+X_{k}^{\prime}}{2}, V_{k}\right) e^{i V_{k} \cdot\left(X_{k}-X_{k}^{\prime}\right)} d V_{k}= \\
& =i \frac{1}{(2 \pi)^{2 d}} \int d u_{1} d u_{2} d w d z d \omega \mathbf{b}\left(2\left|u_{2}\right|, \omega \cdot \frac{u_{2}}{\left|u_{2}\right|}\right) e^{i u_{1} \cdot w} e^{-i u_{2} \cdot z} \times \\
& \quad \times \gamma^{(k+1)}\left(x_{1}-\frac{w+z}{4}, X_{2: k}, \frac{x_{1}+x_{1}^{\prime}}{2}-\frac{w-z}{4}\right. \\
& \left.x_{1}^{\prime}+\frac{w+z}{4}, X_{2: k}^{\prime}, \frac{x_{1}+x_{1}^{\prime}}{2}+\frac{w-z}{4}\right)
\end{aligned}
$$

Recall that

$$
\hat{\mathbf{b}}^{\omega}(\xi)=\int_{\mathbb{R}^{d}} \mathbf{b}\left(|u|, \omega \cdot \frac{u}{|u|}\right) e^{-i u \cdot \xi} d u
$$

Then we have

$$
\begin{aligned}
& i \int C_{1, k+1}^{-} f^{(k+1)}\left(\frac{X_{k}+X_{k}^{\prime}}{2}, V_{k}\right) e^{i V_{k} \cdot\left(X_{k}-X_{k}^{\prime}\right)} d V_{k}= \\
& =\frac{i}{2^{2 d} \pi^{d}} \int_{\mathbb{S}^{d-1}} d \omega \int_{\mathbb{R}^{d}} d z \hat{\mathbf{b}}^{\omega}\left(\frac{z}{2}\right) \times \\
& \quad \times \gamma^{(k+1)}\left(x_{1}-\frac{z}{4}, X_{2: k}, \frac{x_{1}+x_{1}^{\prime}}{2}+\frac{z}{4}, x_{1}^{\prime}+\frac{z}{4}, X_{2: k}^{\prime}, \frac{x_{1}+x_{1}^{\prime}}{2}-\frac{z}{4}\right)
\end{aligned}
$$


Now the gain term.

$$
\begin{aligned}
& i \int C_{1, k+1}^{+} f^{(k+1)}\left(\frac{X_{k}+X_{k}^{\prime}}{2}, V_{k}\right) e^{i V_{k} \cdot\left(X_{k}-X_{k}^{\prime}\right)} d V_{k}= \\
& =i \int d V_{k+1} d \omega \mathbf{b}\left(\left|v_{k+1}-v_{1}\right|, \omega \cdot \frac{v_{k+1}-v_{1}}{\left|v_{k+1}-v_{1}\right|}\right) e^{i V_{k} \cdot\left(X_{k}-X_{k}^{\prime}\right)} \times \\
& \times f^{(k+1)}\left(\frac{x_{1}+x_{1}^{\prime}}{2}, \frac{X_{2: k}+X_{2: k}^{\prime}}{2}, \frac{x_{1}+x_{1}^{\prime}}{2}, v_{1}^{*}, V_{2: k}, v_{k+1}^{*}\right) \\
& =i \frac{1}{(2 \pi)^{d(k+1)}} \int d V_{k+1} d Y_{k+1} d \omega \mathbf{b}\left(\left|v_{k+1}-v_{1}\right|, \omega \cdot \frac{v_{k+1}-v_{1}}{\left|v_{k+1}-v_{1}\right|}\right) \times \\
& \times e^{i V_{k} \cdot\left(X_{k}-X_{k}^{\prime}\right)} e^{-i V_{2: k} \cdot Y_{2: k}} e^{-i\left(v_{1}+P_{\omega}\left(v_{k+1}-v_{1}\right)\right) \cdot y_{1}} e^{-i\left(v_{k+1}-P_{\omega}\left(v_{k+1}-v_{1}\right)\right) \cdot y_{k+1}} \times \\
& \times \gamma^{(k+1)}\left(\frac{x_{1}+x_{1}^{\prime}+y_{1}}{2}, \frac{X_{2: k}+X_{2: k}^{\prime}+Y_{2: k}}{2}, \frac{x_{1}+x_{1}^{\prime}+y_{k+1}}{2},\right. \\
& \left.\frac{x_{1}+x_{1}^{\prime}-y_{1}}{2}, \frac{X_{2: k}+X_{2: k}^{\prime}-Y_{2: k}}{2}, \frac{x_{1}+x_{1}^{\prime}-y_{k+1}}{2}\right) \\
& =i \frac{1}{(2 \pi)^{2 d}} \int d v_{1} d v_{k+1} d y_{1} d y_{k+1} d \omega \mathbf{b}\left(\left|v_{k+1}-v_{1}\right|, \omega \cdot \frac{v_{k+1}-v_{1}}{\left|v_{k+1}-v_{1}\right|}\right) \times \\
& \times e^{i v_{1} \cdot\left(x_{1}-x_{1}^{\prime}\right)} e^{-i\left(v_{1}+P_{\omega}\left(v_{k+1}-v_{1}\right)\right) \cdot y_{1}} e^{-i\left(v_{k+1}-P_{\omega}\left(v_{k+1}-v_{1}\right)\right) \cdot y_{k+1}} \times \\
& \times \gamma^{(k+1)}\left(\frac{x_{1}+x_{1}^{\prime}+y_{1}}{2}, X_{2: k}, \frac{x_{1}+x_{1}^{\prime}+y_{k+1}}{2},\right. \\
& \left.\frac{x_{1}+x_{1}^{\prime}-y_{1}}{2}, X_{2: k}^{\prime}, \frac{x_{1}+x_{1}^{\prime}-y_{k+1}}{2}\right)
\end{aligned}
$$

Let $u_{1}=\frac{1}{2}\left(v_{k+1}+v_{1}\right), u_{2}=\frac{1}{2}\left(v_{k+1}-v_{1}\right)$.

$$
\begin{aligned}
& i \int C_{1, k+1}^{+} f^{(k+1)}\left(\frac{X_{k}+X_{k}^{\prime}}{2}, V_{k}\right) e^{i V_{k} \cdot\left(X_{k}-X_{k}^{\prime}\right)} d V_{k}= \\
& =i \frac{1}{2^{d} \pi^{2 d}} \int d u_{1} d u_{2} d y_{1} d y_{k+1} d \omega \mathbf{b}\left(2\left|u_{2}\right|, \omega \cdot \frac{u_{2}}{\left|u_{2}\right|}\right) \times \\
& \quad \times e^{i u_{1} \cdot\left(x_{1}-x_{1}^{\prime}-y_{1}-y_{k+1}\right)} e^{-i u_{2} \cdot\left(x_{1}-x_{1}^{\prime}-R_{\omega}\left(y_{1}-y_{k+1}\right)\right)} \times \\
& \quad \times \gamma^{(k+1)}\left(\frac{x_{1}+x_{1}^{\prime}+y_{1}}{2}, X_{2: k}, \frac{x_{1}+x_{1}^{\prime}+y_{k+1}}{2},\right. \\
& \left.\frac{x_{1}+x_{1}^{\prime}-y_{1}}{2}, X_{2: k}^{\prime}, \frac{x_{1}+x_{1}^{\prime}-y_{k+1}}{2}\right)
\end{aligned}
$$


Let $w=x_{1}-x_{1}^{\prime}-y_{1}-y_{k+1}, z=x_{1}-x_{1}^{\prime}-R_{\omega}\left(y_{1}-y_{k+1}\right)$.

$$
\begin{aligned}
& i \int C_{1, k+1}^{+} f^{(k+1)}\left(\frac{X_{k}+X_{k}^{\prime}}{2}, V_{k}\right) e^{i V_{k} \cdot\left(X_{k}-X_{k}^{\prime}\right)} d V_{k}= \\
& =i \frac{1}{(2 \pi)^{2 d}} \int d u_{1} d u_{2} d w d z d \omega \mathbf{b}\left(2\left|u_{2}\right|, \omega \cdot \frac{u_{2}}{\left|u_{2}\right|}\right) e^{i u_{1} \cdot w} e^{-i u_{2} \cdot z} \times \\
& \times \gamma^{(k+1)}\left(x_{1}-\frac{1}{2} P_{\omega}\left(x_{1}-x_{1}^{\prime}\right)-\frac{w+R_{\omega}(z)}{4}, X_{2: k},\right. \\
& \frac{x_{1}+x_{1}^{\prime}}{2}+\frac{1}{2} P_{\omega}\left(x_{1}-x_{1}^{\prime}\right)-\frac{w-R_{\omega}(z)}{4}, \\
& x_{1}^{\prime}+\frac{1}{2} P_{\omega}\left(x_{1}-x_{1}^{\prime}\right)+\frac{w+R_{\omega}(z)}{4}, X_{2: k}^{\prime}, \\
& \left.\frac{x_{1}+x_{1}^{\prime}}{2}-\frac{1}{2} P_{\omega}\left(x_{1}-x_{1}^{\prime}\right)+\frac{w-R_{\omega}(z)}{4}\right)
\end{aligned}
$$

We have

$$
\begin{aligned}
& i \int C_{1, k+1}^{+} f^{(k+1)}\left(\frac{X_{k}+X_{k}^{\prime}}{2}, V_{k}\right) e^{i V_{k} \cdot\left(X_{k}-X_{k}^{\prime}\right)} d V_{k}= \\
& =\frac{i}{2^{2 d} \pi^{d}} \int_{\mathbb{S}^{d-1}} d \omega \int_{\mathbb{R}^{d}} d z \hat{\mathbf{b}}^{\omega}\left(\frac{z}{2}\right) \times \\
& \times \gamma^{(k+1)}\left(x_{1}-\frac{1}{2} P_{\omega}\left(x_{1}-x_{1}^{\prime}\right)-\frac{R_{\omega}(z)}{4}, X_{2: k},\right. \\
& \frac{x_{1}+x_{1}^{\prime}}{2}+\frac{1}{2} P_{\omega}\left(x_{1}-x_{1}^{\prime}\right)+\frac{R_{\omega}(z)}{4}, \\
& x_{1}^{\prime}+\frac{1}{2} P_{\omega}\left(x_{1}-x_{1}^{\prime}\right)+\frac{R_{\omega}(z)}{4}, X_{2: k}^{\prime}, \\
& \left.\frac{x_{1}+x_{1}^{\prime}}{2}-\frac{1}{2} P_{\omega}\left(x_{1}-x_{1}^{\prime}\right)-\frac{R_{\omega}(z)}{4}\right)
\end{aligned}
$$

We now turn to the Boltzmann equation. Here it suffices to notice that a solution $f$ of the Boltzmann equation is just a factorized solution of the Boltzmann hierarchy, i.e. $f^{(k)}=f^{\otimes k}$. Using Proposition A.1 and Proposition A.2, we obtain:

Corollary A.3. Let $f \in L^{1}\left([0, T], L^{2}\left(\mathbb{R}^{d} \times \mathbb{R}^{d}\right)\right)$ and let $\gamma$ denote the inverse Wigner transform of $f$. Then if

$$
\left(\partial_{t}+v \cdot \nabla_{x}\right) f=g
$$

holds in the sense of distributions, then we have

$$
\left(i \partial_{t}+\frac{1}{2}\left(\Delta_{x}-\Delta_{x^{\prime}}\right)\right) \gamma=i \int_{\mathbb{R}^{d}} g\left(t, \frac{x+x^{\prime}}{2}, v\right) e^{i v \cdot\left(x-x^{\prime}\right)} d v
$$

in the sense of distributions. 
Corollary A.4. Let $f(x, v)$ be a Schwartz function, and let $\gamma$ denote its inverse Wigner transform. Then

$$
\begin{aligned}
& i \int Q(f, f)\left(\frac{x+x^{\prime}}{2}, v\right) e^{i v \cdot\left(x-x^{\prime}\right)} d v= \\
& =\frac{i}{2^{2 d} \pi^{d}} \int_{\mathbb{S}^{d-1}} d \omega \int_{\mathbb{R}^{d}} d z \hat{\mathbf{b}}^{\omega}\left(\frac{z}{2}\right) \times \\
& \quad \times\left\{\gamma\left(x-\frac{1}{2} P_{\omega}\left(x-x^{\prime}\right)-\frac{R_{\omega}(z)}{4}, x^{\prime}+\frac{1}{2} P_{\omega}\left(x-x^{\prime}\right)+\frac{R_{\omega}(z)}{4}\right) \times\right. \\
& \quad \times \gamma\left(\frac{x+x^{\prime}}{2}+\frac{1}{2} P_{\omega}\left(x-x^{\prime}\right)+\frac{R_{\omega}(z)}{4}, \frac{x+x^{\prime}}{2}-\frac{1}{2} P_{\omega}\left(x-x^{\prime}\right)-\frac{R_{\omega}(z)}{4}\right) \\
& \left.\quad-\gamma\left(x-\frac{z}{4}, x^{\prime}+\frac{z}{4}\right) \gamma\left(\frac{x+x^{\prime}}{2}+\frac{z}{4}, \frac{x+x^{\prime}}{2}-\frac{z}{4}\right)\right\}
\end{aligned}
$$

\section{REFERENCES}

[1] R. Alexandre, Y. Morimoto, S. Ukai, C.-J. Xu, and T. Yang, Global existence and full regularity of the Boltzmann equation without angular cutoff, Comm. Math. Phys. 304 (2011), no. 2, 513-581.

[2] - Local existence with mild regularity for the Boltzmann equation, Kinetic and Related Models 6 (2013), no. 4, 1011-1041.

[3] D. Arsenio, On the global existence of mild solutions to the Boltzmann equation for small data in $L^{D}$, Comm. Math. Phys. 302 (2011), no. 2, 453-476.

[4] C. Cercignani, R. Illner, and M. Pulvirenti, The mathematical theory of dilute gases, Springer Verlag, 1994.

[5] T. Chen and N. Pavlović, On the Cauchy problem for focusing and defocusing GrossPitaevskii hierarchies, Discr. Contin. Dyn. Syst. A 27 (2010), no. 2, 715-739.

[6] R. J. DiPerna and P.-L. Lions, On the Cauchy problem for Boltzmann equations: Global existence and weak stability, Ann. Math. 130 (1989), no. 2, 321-366.

[7] Renjun Duan, On the Cauchy problem for the Boltzmann equation in the whole space: Global existence and uniform stability in $L_{\xi}^{2}\left(H_{X}^{N}\right)$, Journal of Differential Equations 244 (2008), no. 12, 3204-3234.

[8] L. Erdös, B. Schlein, and H.-T. Yau, Derivation of the Gross-Pitaevskii hierarchy for the dynamics of Bose-Einstin condensate, Comm. Pure Appl. Math. 59 (2006), no. $12,1659-1741$.

[9] _ Derivation of the cubic non-linear Schrödinger equation from quantum dynamics of many-body systems, Invent. math. 167 (2007), 515-614.

[10] L. Erdös and H.-T. Yau, Derivation of the nonlinear Schrödinger equation form a many body Coulomb system, Adv. Theor. Math. Phys. 5 (2001), 1169-1205.

[11] I. Gallagher, L. Saint-Raymond, and B. Texier, From Newton to Boltzmann: Hard spheres and short-range potentials, Zurich Lec. Adv. Math. (2014).

[12] P. T. Gressman and R. M. Strain, Global classical solutions of the Boltzmann equation without angular cut-off, J. Amer. Math. Soc. 24 (2011), no. 3, 771-847.

[13] Y. Guo, Classical solutions to the Boltzmann equation for molecules with an angular cutoff, Archive for Rational Mechanics and Analysis 169 (2003), no. 4, 305-353.

[14] E. Hewitt and L. J. Savage, Symmetric measures on Cartesian products, Trans. Amer. Math. Soc. 80 (1955), 470-501.

[15] S. Kaniel and M. Shinbrot, The Boltzmann equation: I. Uniqueness and local existence, Communications in Mathematical Physics 58 (1978), no. 1, 65-84. 
[16] F. King, BBGKY hierarchy for positive potentials, Ph.D. Thesis, 1975.

[17] S. Klainerman and M. Machedon, On the uniqueness of solutions to the GrossPitaevskii hierarchy, Comm. Math. Phys. 279 (2008), no. 1, 169-185.

[18] O. E. Lanford, Time evolution of large classical systems, Dynamical systems, theory and applications, 1975, pp. 1-111.

[19] S. Ukai, On the existence of global solutions of mixed problem for the non-linear Boltzmann equation, Proc. Japan Acad. 50 (1974), no. 3, 179-184.

[20] S. Ukai and T. Yang, The Boltzmann equation in the space $L^{2} \cap L_{\beta}^{\infty}$ : Global and time-periodic solutions, Analysis and Applications 4, no. 3, 263-310. 